\title{
Intramolecular 1,8-Hydrogen Atom Transfer. Stereoselectivity of the Hexopyranos-5' -yl Radical Reactions in Hexp-(1f4)-Hexp Disaccharide Systems
}

\author{
Ángeles Mart'in,* Ine's Pe'rez-Mart'in, Lu'is M. Quintanal, and Ernesto Sua'rez* \\ Instituto de Productos Naturales y Agrobiolog'la del C.S.I.C., Carretera de La Esperanza 3, \\ 38206 La Laguna, Tenerife, Spain \\ angelesmartin@ipna.csic.es; esuarez@ipna.csic.es \\ ReceiVed July 8, 2008
}

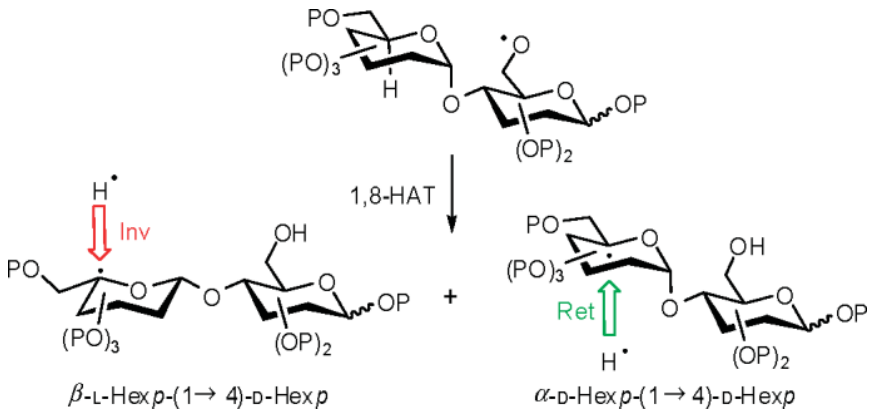

The stereoselective reduction of hexopyranos-5'-yl radicals in R-D-Hexp-(1f4)-D-Hexp disaccharide models is described. These radicals are generated from a 6- $O$-yl radical located in the other monosaccharic unit through a 1,8-hydrogen atom transfer. The reaction, which is strongly influenced by steric and stereoelectronic effects, permits in some cases the transformation of R-D-Hexp-(1f4)-D-Hexp into $\S$-LHexp-(1f4)-D-Hexp disaccharides in a single step with high diastereoselectivity.

\section{Introduction}

The conformational aspects of hexopyranos-1-yl radicals and the stereoselectivity of their reactions have been extensively studied in recent years. ${ }^{1}$ It was found that the conformation of these radicals depends critically on the electronegativity and stereochemistry of the 2-oxygenated substituent. ${ }^{2}$ Thus, mannopyranos-1-yl radicals (I) retain the ${ }^{4} C_{1}$ chair of their precursor 1, while glucopyranos-1-yl radicals (II) exist preferentially in a $B_{2,5}$ boat conformation (Scheme 1). Both radicals, with the 2-oxygenated substituent axially disposed, show a high propensity for quenching along the R-axial direction. This stereoselectivity is caused by two main stereoelectronic effects that have been termed the radical anomeric effect and the quasihomo-anomeric effect [\$-oxygen effect.$^{3}$

(1)(a) Renaud, P. In Radicals in Organic Synthesis; Renaud, P., Sibi, M. P., Eds.; Wiley-VCH: Weinheim, 2001; Vol. 1, pp 400-415. (b) Praly, J.-P. AdV. Carbohydr. Chem. Biochem. 2000, 56, 65-151. (c) Curran, P. C.; Porter, N. A.; Giese, B. Stereochemistry of Radical Reactions: Concepts, Guidelines, and Synthetic Applications; VCH: Weinheim, 1996; pp 131-135. (d) Giese, B. Angew. Chem., Int. Ed. Engl. 1989, 28, 969-980.

(2) (a) Giese, B.; Dupuis, J.; Leising, M.; Nix, M.; Lindner, H. J. Carbohydr. Res. 1987, 171, 329-341. (b) Dupuis, J.; Giese, B.; Rüegge, D.; Fischer, H.; Korth, H.-G.; Sustmann, R. Angew. Chem., Int. Ed. Engl. 1984, 23, 896-898.
Less is known about the stereoselectivity of hexopyranos-5yl radical reactions and detailed studies have not been reported so far except for a few examples. ${ }^{4}$ These radicals with a pseudo$C$-glycoside structure appear to be in a similar situation with regard to the pyranose ring conformation and stereoelectronic stabilization effects. Indeed, the galactopyranos-5-yl radical (III) with an axial oxygenated substituent at C-4 remains in the ${ }^{4} C_{1}$ chair of its precursor $\mathbf{2}$, while the glucopyranos-5-yl radical(IV) adopts a $B_{1,4}$ boat conformation as determined by ESR spectroscopy (Scheme 1)..$^{5}$ In consequence, we should expect a preponderant formation of R-axial substitution products. This seems to be especially the case for the two examples known

(3) (a) Beckwith, A. L. J.; Duggan, P. J. Tetrahedron 1998, 54, 4623-4632. (b) Beckwith, A. L. J.; Duggan, P. J. Tetrahedron 1998, 54, 6919-6928. (c) Korth, H.-G.; Sustmann, R.; Dupuis, J.; Giese, B. J. Chem. Soc., Perkin Trans. 2 1986, 1453-1459. (d) Barton, D. H. R.; Hartwig, W.; Motherwell, W. B. J. Chem. Soc., Chem. Commun. 1982, 4397-4410.

(4) For a recent review on radicals in carbohydrate chemistry, see: (a) Pearce, A. J.; Mallet, J.-M.; Sinay”, P. In Radicals in Organic Synthesis; Renaud, P.; Sibi, M. P., Eds.; Wiley-VCH: Weinheim, 2001; Vol. 2, pp 538-577. (b) Sowa, C. E.; Thiem, J. Angew. Chem., Int. Ed. Engl. 1994, 33, 1979-1981. (c) Korth, H.-G.; Sustmann, R.; Gröninger, K. S.; Leisung, M.; Giese, B. J. Org. Chem. 1988, 53, 4364-4369. (d) Blattner, R.; Ferrier, R. J.; Renner, R. J. Chem. Soc., Chem. Commun. 1987, 1007-1008.

(5) Korth, H.-G.; Praly, J.-P.; Somsa'k, L.; Sustmann, R. Chem. Ber. 1990, $123,1155-1160$. 


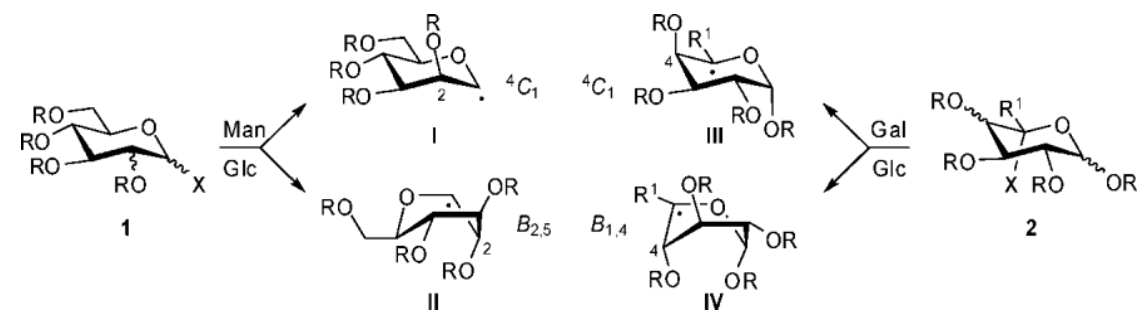

$\left.{ }^{a} \mathrm{R}\right) \quad$ protective group; $\mathrm{R}^{1}$ ) see text; $\mathrm{X}$ ) $\mathrm{Br}$.

where the $n-\mathrm{Bu}_{3} \mathrm{SnH}$ reduction of $(5 S)-1,2,3,4$-tetra- $O$-acetyl5-bromo-8-cyano-6,7,8-trideoxy-§-D-xylo-octopyranose $(2, \mathrm{R})$ Ac; $\left.\left.\left.\mathrm{R}^{1}\right) \quad\left(\mathrm{CH}_{2}\right)_{2} \mathrm{CN} ; \mathrm{X}\right) \quad \mathrm{Br}\right)^{4 \mathrm{~d}}$ and $(5 \mathrm{~S})-1,2,3,4,6$-penta- $O$ acetyl-5-bromo-R-D-xylo-hexopyranose ( $\left.2, \mathrm{R}) \quad \mathrm{Ac} ; \mathrm{R}^{1}\right)$ $\mathrm{CH}_{2} \mathrm{OAc}, \mathrm{X}$ ) $\left.\mathrm{Br}\right)^{4 \mathrm{c}}$ apparently proceed by axial quenching with total retention of configuration.

The reduction of methyl (5R)-1,2,3,4-tetra- $O$-acetyl-5-bromoD-xylo-hexopyranuronate ( $\left.\left.2, \mathrm{R}) \quad \mathrm{Ac} ; \mathrm{R}^{1}\right) \quad \mathrm{CO}_{2} \mathrm{Me} ; \mathrm{X}\right) \mathrm{Br}$ ) has been studied in more detail. ${ }^{6}$ The stereochemistry of the reaction seems to be dependent on the temperature and/or concentration of the tin hydride as well as on the configuration of the substituent at $\mathrm{C}-1 .{ }^{6 c}$ Surprisingly, the products were obtained with diastereoselectivities up to $3: 1$ in favor of the inverted L-iduronic acid derivative. At first sight, this is probably attributable to a radical stabilization by the carboxylate group, which permits ring inversion to a ${ }^{1} C_{4}$ chair and stannane quenching along the $\S$-axial direction. This apparently anomalous behavior has attracted some preparative interest because L-iduronic acid is a key constituent of heparin and other related glycosaminoglycans and is essential for the construction of heparin-based oligosaccharides. ${ }^{7}$

\section{Results and Discussion}

In a previous paper from this laboratory we have described an example of 1,8-hydrogen atom transfer (HAT) between the two glucopyranose units of a heptamethylated R-D-Glc $p$-(1f4)$\S$-D-Glcp disaccharide (§-maltose) $(3)^{8}$ through a ninemembered transition state (Scheme 2). ${ }^{9}$ The hydrogen abstraction from $\mathrm{C}-5^{\prime}$ is promoted, in a highly efficient and completely regioselective manner, by the 6-O-yl radical $(\mathbf{V})$ generated in situ from reaction of the 6-O-phthalimide derivative 4 with $n$ $\mathrm{Bu}_{3} \mathrm{SnH} / \mathrm{AIBN}$. In this case the $C$-radical $(\mathbf{V I})$ reduction gave also predominant inversion of the configuration at C-5' and consequent transformation of this D-glucose moiety in L-idose (L-Ido/D-Glc, 1.6:1) (Table 1, entry 1). After some experimenta-

(6) For a review on the preparation of L-iduronic synthons, see: (a) Pellissier, H. Org. Prep. Proced. Int. 2002, 34, 441-465. (b) Yu, H. N.; Furukawa, J.-I. Ikeda, T.; Wong, C.-H. Org. Lett. 2004, 6, 723-726. (c) Medakovic, D. Carbohydr. Res. 1994, 253, 299-300. (d) Chiba, T.; Sinaÿ, P. Carbohydr. Res. 1986, 151, 379-389. (e) Blattner, R.; Ferrier, R. J. J. Chem. Soc., Perkin Trans. 1 1980, 1523-1527. (f) Ferrier, R. J.; Tyler, P. C. J. Chem. Soc., Perkin Trans. 1 1980, 1528-1534.

(7) (a) Noti, C.; de Paz, J. L.; Polito, L.; Seeberger, P. H. Chem. Eur. J. 2006, 12, 8664-8686. (b) Zhou, Y.; Lin, F.; Chen, J.; Yu, B. Carbohydr. Res. 2006, 341, 1619-1629. (c) de Paz, J. L.; Mart'in-Lomas, M. Eur. J. Org. Chem. 2005, 1849-1858. (d) Lubineau, A.; Lortat-Jacob, H.; Gavard, O.; Sarrazin, S.; Bonnaffe', D. Chem. Eur. J. 2004, 10, 4265-4282. (e) Lohman, G. J. S.; Seeberger, P. H. J. Org. Chem. 2004, 69, 4081-4093. (f) Bindscha"dler, P.; Noti, C.; Castagnetti, E.; Seeberger, P. H. HelV. Chim. Acta 2003, 89, 2591-2610. (8) (a) Aspinall, G. O.; Igarashi, O.; Krishnamurthy, T. N.; Mitura, W.; Funabashi, M. Can. J. Chem. 1976, 54, 1708-1713. (b) Aspinall, G. O.; Krishnamurthy, T. N.; Mitura, W.; Funabashi, M. Can. J. Chem. 1975, 53, 21822188.

(9) Francisco, C. G.; Herrera, A. J.; Kennedy, A. R.; Melia'n, D.; Sua'rez, E. Angew. Chem., Int. Ed. 2002, 41, 856-858.
SCHEME 2. Reduction of Hexopyranos-5'-yl Radicals in r-D-GIcp-(1f4)- $\beta$-D-GIcp Disaccharides ${ }^{a}$
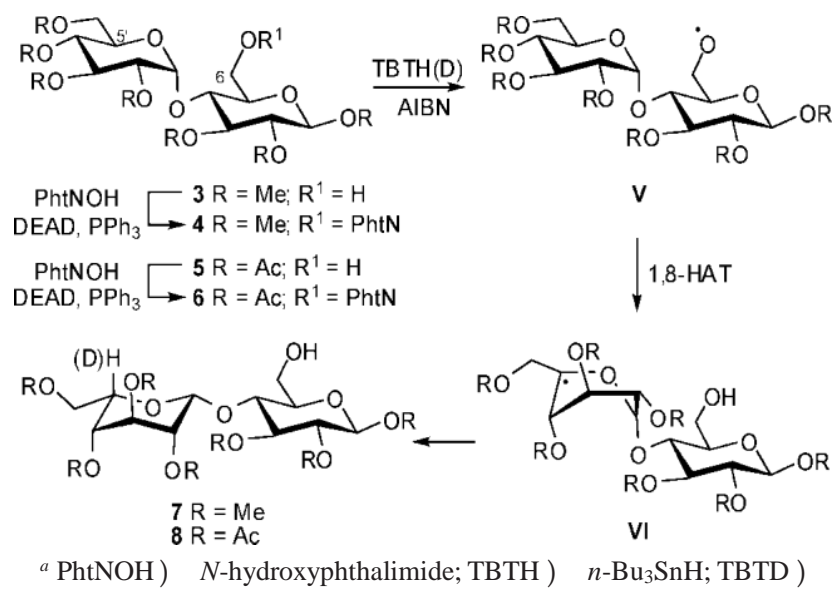
$n-\mathrm{Bu}_{3} \mathrm{SnD}$.

tion, we have now found that this stereoselectivity can be improved up to a inversion/retention ratio of $2.5: 1$ by slowing down the stannane quenching reducing the amount and concentration of tin hydride (entry 2).

This last result, which suggests that 1,8-HAT may be a synthetically useful protocol for the inversion of configuration at $\mathrm{C}^{-5^{\prime}}$ and consequent transformation of this $\S$-maltose derivative 4 into a $\S$-L-Ido $p$-(1f4)- $\$-D-G l c p$ system (7) in a single step, encouraged a further exploratory effort with variously structured disaccharide substrates. ${ }^{10}$

It has been suggested that the quasi-homo-anomeric effect increases with the increase in electronegativity of the $\S$-oxygenated substituent ${ }^{2 \mathrm{~b}}$ so we decided to prepare the heptaacetylated 6- $O$-phthalimide ${ }^{11}$ derivative 6 from the alcohol 5 and $N$ -

hydroxyphthalimide under Mitsunobu conditions. ${ }^{12}$ To our delight, the HAT reaction afforded the inverted product $\mathbf{8}$ with excellent diastereoselectivity $(\mathbf{8} / \mathbf{5}, 14: 1)$ (entry 3$)$. In these aldose systems, as in the uronate system, a mechanism of $\S$-axial radical quenching on the inverted ${ }^{1} C_{4}$ chair could also be operating.

In order to calculate correct diastereoselectiveratios deuterium incorporation studies using $n-\mathrm{Bu}_{3} \mathrm{SnD} / \mathrm{AIBN}$ as reagent were

carried out to discriminate between deuterated alcohol $\mathbf{5}$ produced by abstraction with retention and the undeuterated one generated by reduction of the alkoxyl radical prior to the abstraction reaction. As expected, complete incorporation of

(10)This disaccharide model is closely related to R-L-Idop-(1f4)-R-D-Glcp, a repetitive unit in heparin glycosaminoglycan.

(11)(a) Kim, S.; Lee, T. A.; Song, Y. Synlett 1998, 471-472. (b) Okada, K.; Okamoto, K.; Oda, M. J. Am. Chem. Soc. 1988, 110, 8736-8738. (c) Okada, K.; Okamoto, K.; Oda, M. J. Chem. Soc., Chem. Commun. 1989, 1636-1637.

(d) Barton, D. H. R.; Blundell, P.; Jaszberenyi, J. Cs. Tetrahedron Lett. 1989, 30, 2341-2344.

(12) (a) Mitsunobu, O. Synthesis 1981, 1-28. (b) Grochowski, E.; Jurczak, J. Synthesis 1976, 682-684. 
TABLE 1. Stereoselective Reduction of Hexopyranos-5' - yl Radicals

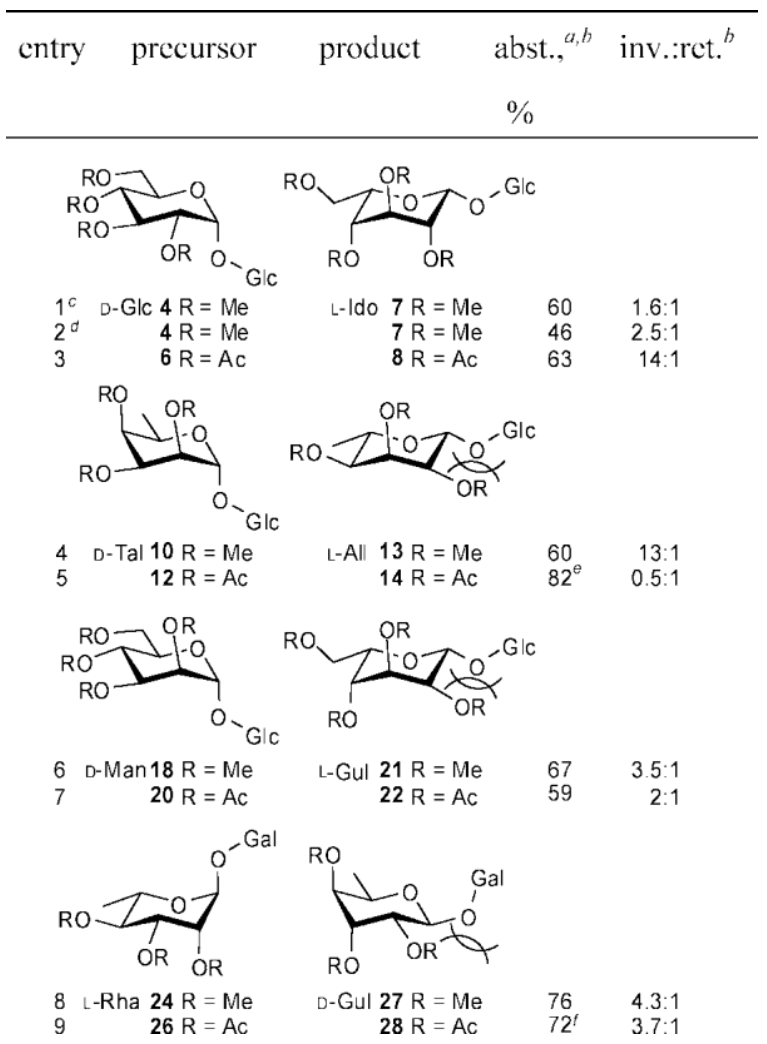

${ }^{a}$ Isolated 1,8-abstraction yield. ${ }^{b}$ The yield of retention product was estimated by $n-\mathrm{Bu}_{3} \mathrm{SnD} / \mathrm{AIBN}$ experiments. ${ }^{c} n-\mathrm{Bu}_{3} \mathrm{SnH}$ ( 9 equiv), AIBN ( 0.15 equiv), $\mathrm{PhH} 1.8 \mathrm{M}$, reflux, 1 h. ${ }^{d} n-\mathrm{Bu}_{3} \mathrm{SnH}$ ( 1 equiv), $\mathrm{AIBN}(0.1$ equiv), $\mathrm{PhH} 0.01 \mathrm{M}$, reflux, $1 \mathrm{~h} .{ }^{e}$ Included retained product $15(22 \%)$. ${ }^{f}$ Included abstraction product $30(5 \%)$.

deuterium at $\mathrm{C}-5^{\prime}$ is detected in the inverted product $\mathbf{8}$ and there is no observable deuterium scrambling at other positions of the molecule within NMR detection limits. The results are summarized in Table 1 and in Supporting Information.

To better understand the scope and stereoselectivity of this C-5' radical stannane quenching, we decided to prepare various disaccharides with the correct relative stereochemistry at the four chiral centers $\left(\mathrm{C}-4, \mathrm{C}-5, \mathrm{C}-\mathrm{1}^{\prime}\right.$, and $\left.\mathrm{C}-5^{\prime}\right)$ involved in the 1,8-HAT reaction: ${ }^{13}$ R-D-Tal $p$-(1f4)-R-D-Glc $p(\mathbf{9}, 11)$, R-DManp-(1f4)-R-D-Glcp (17, 19), and R-L-Rhap-(1f4)-R-D-Gal $p$ $(\mathbf{2 3}, \mathbf{2 5})$ (Schemes 3-5). Compounds 9 and 17 were effectively synthesized by TMSOTf-mediated glycosylation of methyl 6- $O$ tert-butyldiphenylsilyl-2,3-di- $O$-methyl-R-D-glucopyranoside, using 2,3,4-tri- $O$-methyl-6-deoxy-R-D-talopyranosyl and 2,3,4,6tetra- $O$-methyl-R-D-mannopyranosyl trichloroacetimidates, respectively, as glycosyl donors. ${ }^{14}$ Analogously, compound 23 (Scheme 5) was synthesized by glycosylation of methyl 6-O-tertbutyldiphenylsilyl-2,3-di- $O$-methyl-R-D-galactopyranoside with 2,3,4-tri- $O$-methyl-R-L-rhamnopyranosyl trichloroacetimidate. ${ }^{15}$ The corresponding acetates $\mathbf{1 1}, \mathbf{1 9}$, and $\mathbf{2 5}$ were prepared by a

(13) Mart'in, A.; Pe'rez-Mart'in, I.; Quintanal, L. M.; Sua'rez, E. Org. Lett. 2007, 9, 1785-1788.

(14)(a) Matsuo, I.; Isomura, M.; Miyazaki, T.; Sakakibara, T.; Ajisaka, K. Carbohydr. Res. 1997, 305, 401-413. (b) Kere'koya'rto', J.; Kamerling, J. P Bouwstra, J. B.; Vliegenthart, J. F. G.; Lipta'k, A. Carbohydr. Res. 1989, 186 51-62. For a review on disaccharide synthesis with trichloroacetimidates, see: (c) Schmidt, R. R.; Jung, K.-H. In PreparatiVe Carbohydrate Chemistry; Hanessian, S., Ed.; Marcel Dekker: New York, 1997; pp 283-312.
SCHEME 3. Reduction of Hexopyranos -5 ' - yl Radicals in r-D-Talp-(1f4)-r-D-Glcp Disaccharides ${ }^{a}$

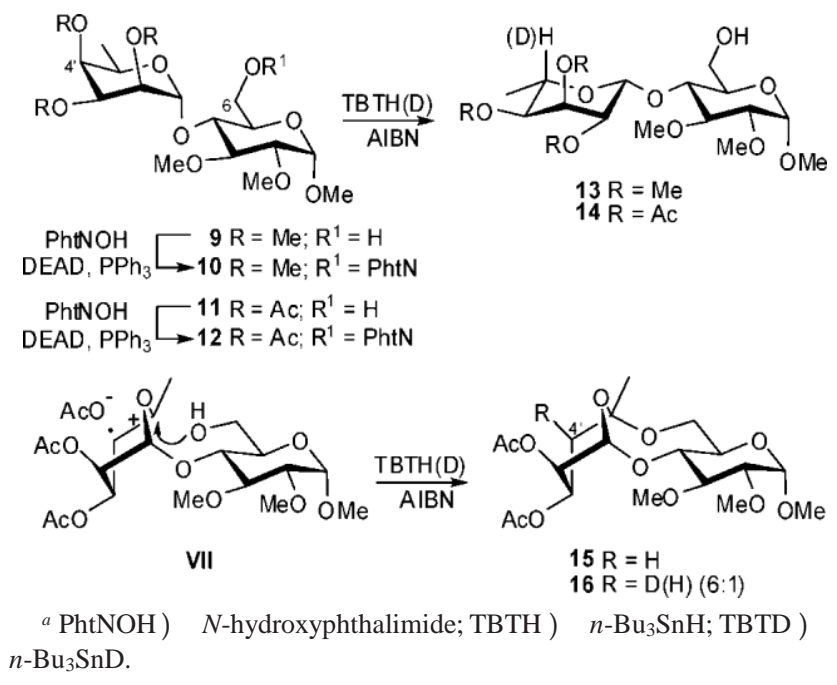

SCHEME 4. Reduction of Hexopyranos-5'-yl Radicals in r-D-Manp-(1f4)-r-D-Glcp Disaccharides ${ }^{a}$

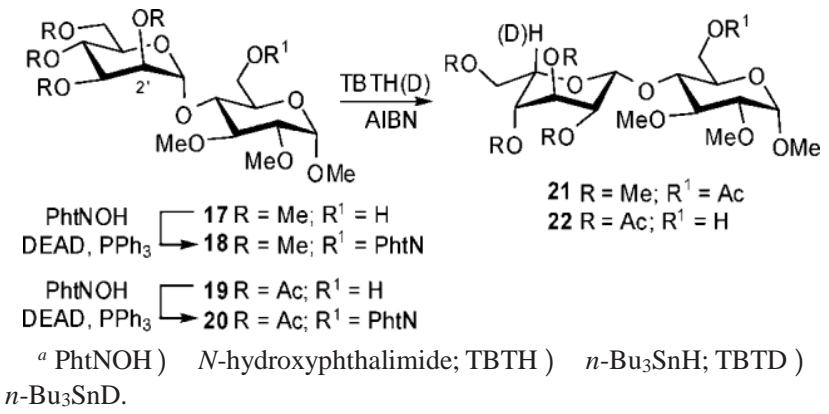

SCHEME 5. Reduction of Hexopyranos-5' - yl Radicals in r-L-Rhap-(1f4)-r-D-Galp Disaccharides ${ }^{a}$

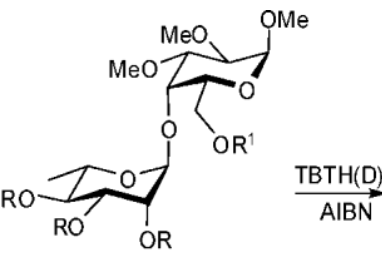
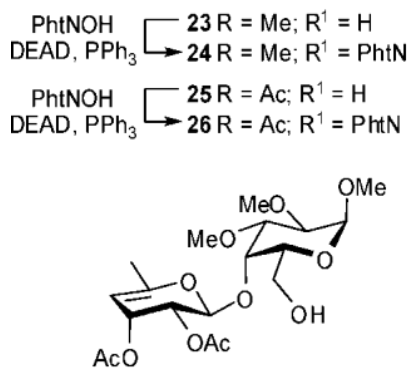

30

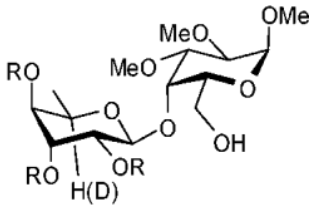

$27 \mathrm{R}=\mathrm{Me}$

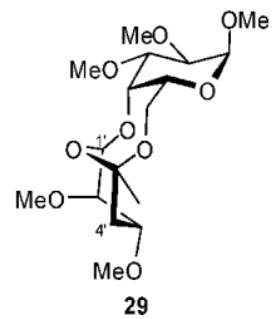

$\left.{ }^{a} \mathrm{PhtNOH}\right) \quad N$-hydroxyphthalimide; TBTH ) $\quad n-\mathrm{Bu}_{3} \mathrm{SnH}$; TBTD ) $n-\mathrm{Bu}_{3} \mathrm{SnD}$

completely analogous synthetic protocol as described in Supporting Information.

The R-D-Tal $p$-(1f4)-R-D-Glc $p$ disaccharide 9 with the Dtalose moiety was selected, because the presence of the three axial substituents was expected to enhance the chair inversion rate (Scheme 3). Indeed, when methylated 6- $O$-phthalimide 10 was treated with $n$-Bu $3 \mathrm{SnH} / \mathrm{AIBN}$ the $\S$-L-All $p$-(1f4)-R-D-Glc $p$ 
disaccharide 13 was obtained in good yield and excellent inversion-retention ratio $(\mathbf{1 3 / 9}, \mathbf{1 3 : 1 )}$ (entry 4). In stark contrast, the 6-O-phthalimide $\mathbf{1 2}$ with the peracetylated D-talose moiety afforded preferentially retained product 11 with modest diastereoselectivity $(\mathbf{1 4} / \mathbf{1 1}, 0.5: 1)$ (entry 5$)$. In this last case the reaction was accompanied by an unexpected side product $\mathbf{1 5}$ in low yield (22\%). The structure and stereochemistry of $\mathbf{1 5}$ with a 4'-deoxygenated carbon and a 1,3,5-trioxocane cycle as principal features were determined by extensive use of ${ }^{1} \mathrm{H}$ and ${ }^{13} \mathrm{C}$ NMR including DEPT, and 2D-COSY, HMBC, and HSQC experiments. When the reaction was carried out with $n-\mathrm{Bu}_{3} \mathrm{SnD}$, compound 16 was obtained with approximately $86 \%$ deuterium incorporation at C-4'. The axial disposition of the deuterium was determined by ${ }^{1} \mathrm{H}$ NMR indicating an effective shielding of the R-pyranose ring by the 1,3,5-trioxocane moiety. ${ }^{16}$ These results suggest that the formation of $\mathbf{1 5}$ takes place by two different mechanisms: (a) a 5'-radical reductive elimination to give a $4^{\prime}$-enol ether and subsequent alcohol addition which is responsible for the minor undeuterated compound (vide infra) and (b) an interesting tandem 1,8-HAT-intramolecular cine substitution mechanism with the $4^{\prime}$-acetate as the leaving group and the oxygen at C-6 acting with an umpolung reactivity during the reaction, first as an electrophilic alkoxyl radical and then as a nucleophile. ${ }^{17}$ The stereochemistry of $\mathbf{1 5}$ suggests that the nucleophilic attack occurred by the R-axial side on the stabilized radical in a ${ }^{4} C_{1}$ chair conformation (VII) with retention of configuration at C-5' (Scheme 3).

Next, we were interested in studying the influence of the stereochemistry of substituents other than C-4'. In this context, disaccharide R-D-Manp-(1f4)-R-D-Glcp (17), which differs from 3 only in the configuration at $\mathrm{C}-2^{\prime}$, was selected (Scheme 4). The reaction of permethylated 6- $O$-phthalimide 18 with $n$ $\mathrm{Bu}_{3} \mathrm{SnH} / \mathrm{AIBN}$ afforded, after acetylation, disaccharide $\S$-LGulp-(1f4)-R-D-Glc $p 21$ with an inversion-retention ratio of $3.5: 1$, which is somewhat higher than that of compound 4 (2.5: 1) (compare entries 6 and 2). On the other hand, compound 22 with an inversion-retention ratio of $2: 1$, substantially lower than in the case of compound $\mathbf{6}(14: 1)$, is obtained during the reaction of tetraacetyl 6-O-phthalimide 20 (compare entries 7 and 3). From the results of these two experiments, it is evident that the stereochemistry of the reduction of the C-5' radical is strongly influenced by both the stereochemistry and nature of the C-2' substituent.

Our next models, the R-L-Rhap-(1f4)-R-D-Galp derivatives ( 23 and 25), may not be so useful from the synthetic point of view (Scheme 5). Indeed, the C-5' inversion should transform the L-rhamnose moiety into a readily accessible 6-deoxy-Dgulose, but we believe them to be important to shed some light on the stereochemistry of the reaction mechanism and further extend the scope of the methodology. In both cases the reaction predominantly produced inverted D-disaccharides (27 and $\mathbf{2 8}$, respectively) with high diastereoselectivity (entries 8 and 9). Contrary to the other examples discussed above during the

(15)(a) Wang, J.; Li, J.; Tuttle, D.; Takemoto, J. Y.; Chang, C.-W. T. Org. Lett. 2002, 4, 3997-4000. (b) van Steijn, A. M. P.; Kamerling, J. P.; Vliegenthart, J. F. G. Carbohydr. Res. 1991, 211, 261-277.

(16) By comparison of the 4-H coupling constant with those of the undeuterated compound 15. Compound 16: $2.03\left(1 \mathrm{H}\right.$, br d, $\left.J_{3^{\prime}, 4^{\prime} \mathrm{eq}}\right) 5.0 \mathrm{~Hz}$, $\left.4^{\prime}-\mathrm{H}_{\mathrm{eq}}\right)$. Compound 15: $\left.\left.1.98\left(1 \mathrm{H}, \mathrm{dd}, J_{g e m}\right) \quad 12.6, J_{3^{\prime}, 4^{\prime} \mathrm{ax}}\right) \quad 12.6 \mathrm{~Hz}, 4^{\prime}-\mathrm{H}_{\mathrm{ax}}\right)$; $\left.\left.2.06\left(1 \mathrm{H}, \mathrm{dd}, J_{\text {gem }}\right) \quad 12.6, J_{3^{\prime}, 4^{\prime} \mathrm{eq}}\right) \quad 4.8 \mathrm{~Hz}, 4^{\prime}-\mathrm{H}_{\mathrm{eq}}\right)$.

(17)For a review, see: (a) Crich, D. In Radicals in Organic Synthesis; Renaud, P., Sibi, M. P., Eds.; Wiley-VCH: Weinheim, 2001; Vol. 2, pp 188-206. For a related 1,5-HAT-intramolecular cine substitution reaction, see: (b) Crich, D.; Huang, X.; Newcomb, M. J. Org. Chem. 2000, 65, 523-529. (c) Crich, D.; Huang, X.; Newcomb, M. Org. Lett. 1999, 1, 225-227. inversion reaction, the pyranose ring undergoes conformational changes from ${ }^{1} C_{4}$ to ${ }^{4} C_{1}$ chair and the radical quenching is now from the R-axial side of the molecule. The formation of two minor products $\mathbf{2 9}$ and $\mathbf{3 0}$ from these reactions deserves a brief comment (Scheme 5). The 4-deoxy-1,3,5-trioxocane 29 was formed in $5 \%$ yield during the reduction of $6-O$-phthalimide 24. In principle, this product is analogous to $\mathbf{1 5}$, but the absence of deuterium incorporation at C-4' when the reaction was carried out using $n$ - $\mathrm{Bu}_{3} \mathrm{SnD}$ suggests that only one mechanism operates in this case and argues in favor of a $5^{\prime}$-radical reductive elimination and subsequent alcohol addition to the $4^{\prime}$-enol ether. The isolation of a small amount of undeuterated 4 '-enol ether 30 during the reaction of $6-O$-phthalimide 26 with $n-\mathrm{Bu}_{3} \mathrm{SnD} /$ AIBN supports the proposed mechanism.

From the study of the results summarized in Table 1 several general conclusions can be drawn. With the $O$-methyl-protected carbohydrates $4, \mathbf{1 0}, \mathbf{1 8}$, and 24 the steric effects clearly govern the stereochemical course of the reaction, whereas stereoelectronic factors appear to be less important (entries 2, 4, 6, and 8 ). The observed inversion/retention ratio is in reasonably good agreement with the expected instability of the ${ }^{4} C_{1}$ chair, and the number of axial substituents in the starting monosaccharide R-D-Tal > R-L-Rha g R-D-Man > R-D-Glc, 13, 4.5, 3.5, and 2.5 , respectively. ${ }^{18}$

On the contrary, it is also clear that stereoelectronic factors are much more important than steric effects in the cases of the

$O$-acetyl-protected carbohydrates $\mathbf{6}$ and $\mathbf{1 2}$. The reaction of acetylated R-D-Glc $\mathbf{6}$ occurs by $\S$-axial radical quenching to give the $\S$-L-Ido derivative $\mathbf{8}$ where the 4 '-acetyl groupis axially disposed, with excellent inversion/retention ratio (entry 3 ). The reaction of acetylated R-D-Tal $\mathbf{1 2}$ is illustrative of the greater

importance of stereoelectronic over steric effects in these esterified carbohydrates, an R-axial radical attack now giving principally retention of configuration at $5^{\prime}$ (entry 5). In striking

contrast the $O$-methyl-R-D-Tal derivative 10, in a relatively unstable ${ }^{4} C_{1}$ conformation and with the $4^{\prime}$-methoxyl group in the axial position, almost exclusively gives inversion (entry 4). In the reaction of the esterified D-Man 20 and L-Rha 26 models, the equatorial-equatorial interaction between the glycosidic bond and the $2^{\prime}$-acetyl substituent seems to be an important source of instability for the inverted product and a substantial decrease in the inversion preference is observed (compare entries 7 and 9 with 3). This effect is not observed in the case of $O$-methyl-D-Man 18 and L-Rha 24 models, perhaps due to the lower steric demand of the methoxyl groups (compare entries 6 and 8 with 2). ${ }^{19}$ We are currently engaged in further studies using more electronegative and more sterically demanding substituents to expand the synthetic utility of this methodology.

\section{Experimental Section}

General Methods. Melting points were determined with a hotstage apparatus. Optical rotations were measured at the sodium line at ambient temperature in $\mathrm{CHCl}_{3}$ solutions. IR spectra were recorded in film unless otherwise stated. NMR spectra were determined at $500 \mathrm{MHz}$ for ${ }^{1} \mathrm{H}$ and $125.7 \mathrm{MHz}$ for ${ }^{13} \mathrm{C}$ in $\mathrm{CDCl}_{3}$ unless otherwise stated, in the presence of TMS as internal standard. Mass spectra were determined at $70 \mathrm{eV}$ unless otherwise stated. Merck silica gel $60 \mathrm{PF}$ (0.063-0.2 mm) was used for column chromatography.

(18) Rao, V. S. R.; Qasba, P. K.; Balaji, P. V.; Chandrasekaran, R. Conformation of Carbohydrates, Harwood Academic: Australia, 1998; pp 49 90 .

(19) Beckwith, A. L. J.; Page, D. M. J. Org. Chem. 1998, 63, 5144-5153. 
Circular layers of $1 \mathrm{~mm}$ of Merck silica gel $60 \mathrm{PF}_{254}$ were used on a Chromatotron for centrifugally assisted chromatography. Commercially available reagents and solvents were analytical grade or were purified by standard procedures prior to use. All reactions involving air- or moisture-sensitive materials were carried out under a nitrogen atmosphere. The spray reagents for TLC analysis were conducted with $0.5 \%$ vanillin in $\mathrm{H}_{2} \mathrm{SO}_{4}$-EtOH (4:1) and further heating until development of color.

Reductive HAT of Methyl 2,3,4,6-Tetra- $O$-methyl-r-D-glucopyranosyl-(1f4)-2,3-di- $O$-methyl-6- $O$-phthalimido-r-D-glucopyranoside (4). Method A with $n-\mathrm{Bu}_{3} \mathrm{SnH}$. A solution of phthalimide $4^{9}(32 \mathrm{mg}, 0.055 \mathrm{mmol})$ in dry benzene $(4.1 \mathrm{~mL})$ containing $n-\mathrm{Bu}_{3} \mathrm{SnH}(15 \mu \mathrm{L}, 0.055 \mathrm{mmol})$ and AIBN (1 mg, 0.006 mmol) was heated at reflux temperature for $1 \mathrm{~h}$. After cooling to room temperature the reaction mixture was concentrated under reduced pressure. The residue was dissolved in $\mathrm{CH}_{3} \mathrm{CN}$, washed with $n$-hexane and the combined more polar extracts were concentrated under reduced pressure. Column chromatography of the crude residue (hexanes-EtOAc, 10:90 f 0:100) afforded methyl 2,3,4,6-tetra- $O$-methyl-R-D-glucopyranosyl-(1f4)-2,3-di$O$-methyl- $\S$-D-glucopyranoside (3) (7 mg, $0.016 \mathrm{mmol}, 29 \%)$ and methyl 2,3,4,6-tetra- $O$-methyl-§-L-idopyranosyl-(1f4)-2,3-di- $O$ methyl-§-D-glucopyranoside (7) (13 mg, $0.030 \mathrm{mmol}, 54 \%)$, both as colorless oils.

Method B with $\boldsymbol{n}$-Bu $\mathbf{B n D}$. A solution of phthalimide $4(25 \mathrm{mg}$, $0.042 \mathrm{mmol})$ in dry benzene $(3.2 \mathrm{~mL})$ containing $n-\mathrm{Bu}_{3} \mathrm{SnD}(12$ $\mu \mathrm{L}, 0.043 \mathrm{mmol})$ and AIBN (1 mg, $0.004 \mathrm{mmol})$ was heated at reflux temperature for $1 \mathrm{~h}$. After this time another portion of $n$ $\mathrm{Bu}_{3} \mathrm{SnD}(12 \mu \mathrm{L}, 0.043 \mathrm{mmol})$ and $\operatorname{AIBN}(1 \mathrm{mg}, 0.004 \mathrm{mmol})$ were added and heating at reflux was continued for an additional $1 \mathrm{~h}$. After cooling to room temperature the reaction mixture was concentrated under reduced pressure. The residue was dissolved in $\mathrm{CH}_{3} \mathrm{CN}$, washed with $n$-hexane and the combined more polar extracts were concentrated under reduced pressure. Column chromatography of the crude residue (hexanes-EtOAc, 10:90 f 0:100) afforded methyl 2,3,4,6-tetra- $O$-methyl-R-D-[5-2 H] glucopyranosyl(1f4)-2,3-di-O-methyl-R-D-glucopyranoside (3-[D]) (7.8 mg, 0.018 mmol, $41 \%,{ }^{1} \mathrm{H} /{ }^{2} \mathrm{H}$ ratio $7: 3$ ) and methyl 2,3,4,6-tetra- $O$-methylR-L-(5-2H)idopyranosyl-(1f4)-2,3-di- $O$-methyl-R-D-glucopyranoside [7-(D)] (6.3 mg, $0.014 \mathrm{mmol}, 33 \%)$, both as colorless oils.

Methyl 2,3,4,6-Tetra- $O$-acetyl-r-D-glucopyranosyl-(1f4)-2,3di- $\boldsymbol{O}$-acetyl-6- $\boldsymbol{O}$-phthalimido- $\beta$-D-glucopyranoside (6). DEAD (50 $\mu \mathrm{L}, 0.306 \mathrm{mmol}$ ) was added dropwise to a stirred solution of methyl 2,3,4,6-tetra- $O$-acetyl-R-D-glucopyranosyl-(1f4)-2,3-di- $O$-acetylR-D-glucopyranoside (5) ${ }^{20}(74.5 \mathrm{mg}, 0.122 \mathrm{mmol}), \mathrm{N}$-hydroxyphthalimide $(50.5 \mathrm{mg}, 0.306 \mathrm{mmol})$ and $\mathrm{PPh}_{3}(79 \mathrm{mg}, 0.306 \mathrm{mmol})$ in dry THF $(1.2 \mathrm{~mL})$ and the resulting solution was stirred at $0{ }^{\circ} \mathrm{C}$ for $1.5 \mathrm{~h}$. Then the solvent was removed and the crude was quenched with water and extracted with ether. The combined extracts were dried over $\mathrm{Na}_{2} \mathrm{SO}_{4}$ and concentrated under reduced pressure. The residue obtained was purified by column chromatography (hexanes-EtO $\mathrm{Et}_{2}, 30: 70$ ) to give compound 6 (70 mg, 0.093 mmol, 76\%) as a white foam: $[\mathrm{R}]_{\mathrm{D}}+76.6(c, 0.325)$; IR $1746 \mathrm{~cm}^{-1}$; ${ }^{1} \mathrm{H}$ NMR $(400 \mathrm{MHz}) \delta_{\mathrm{H}} 1.983(3 \mathrm{H}, \mathrm{s}), 1.986(3 \mathrm{H}, \mathrm{s}), 2.00(3 \mathrm{H}, \mathrm{s})$, $2.026(3 \mathrm{H}, \mathrm{s}), 2.035(3 \mathrm{H}, \mathrm{s}), 2.08(3 \mathrm{H}, \mathrm{s}), 3.19(3 \mathrm{H}, \mathrm{s}), 3.82(1 \mathrm{H}$, ddd, $J) \quad 9.8,4.5,1.6 \mathrm{~Hz}), 4.03-4.09(2 \mathrm{H}, \mathrm{m}), 4.28(1 \mathrm{H}, \mathrm{dd}, J)$ 12.7, $4.5 \mathrm{~Hz}), 4.39(1 \mathrm{H}, \mathrm{dd}, J) \quad 7.9,4.5 \mathrm{~Hz}), 4.42(1 \mathrm{H}, \mathrm{d}, J) 9.0$ $\mathrm{Hz}), 4.46(1 \mathrm{H}, \mathrm{dd}, J) \quad 13.0,4.8 \mathrm{~Hz}), 4.58(1 \mathrm{H}, \mathrm{dd}, J) \quad 13.0,1.6$ $\mathrm{Hz}), 4.80(1 \mathrm{H}, \mathrm{dd}, J) \quad 9.3,7.7 \mathrm{~Hz}), 4.87(1 \mathrm{H}, \mathrm{dd}, J) \quad 10.6,4.0$ $\mathrm{Hz}), 5.03(1 \mathrm{H}, \mathrm{dd}, J) \quad 9.8,9.8 \mathrm{~Hz}), 5.24(1 \mathrm{H}, \mathrm{dd}, J) \quad 9.0,9.0$ $\mathrm{Hz}), 5.35(1 \mathrm{H}, \mathrm{dd}, J) \quad 10.6,9.8 \mathrm{~Hz}), 5.45(1 \mathrm{H}, \mathrm{d}, J) \quad 4.0 \mathrm{~Hz})$, 7.71-7.75 (2H, m), 7.79-7.83 (2H, m); ${ }^{13} \mathrm{C} \mathrm{NMR} \mathrm{(100.6} \mathrm{MHz)}$ $\delta_{\mathrm{C}} 20.5\left(\mathrm{CH}_{3}\right), 20.56\left(2 \times \mathrm{CH}_{3}\right), 20.62\left(2 \times \mathrm{CH}_{3}\right), 20.9\left(\mathrm{CH}_{3}\right)$, $56.3\left(\mathrm{CH}_{3}\right), 61.9\left(\mathrm{CH}_{2}\right), 68.1(\mathrm{CH}), 68.5(\mathrm{CH}), 69.5(\mathrm{CH}), 70.1$ $(\mathrm{CH}), 71.7(\mathrm{CH}), 71.8(\mathrm{CH}), 73.8(\mathrm{CH}), 75.2(\mathrm{CH}), 75.4\left(\mathrm{CH}_{2}\right)$,

(20) (a) Bock, K.; Pedersen, H. Acta Chem. Scand. Ser. B 1988, 42, 75-85. (b) Cottaz, S.; Apparau, C.; Driguez, H. J. Chem. Soc., Perkin Trans. 11991 2235-2241.
95.5 $(\mathrm{CH}), 101.0(\mathrm{CH}), 123.5(2 \times \mathrm{CH}), 128.8(2 \times \mathrm{C}), 134.5(2$ $\times \mathrm{CH}), 163.1(2 \times \mathrm{C}), 169.5(\mathrm{C}), 169.6(\mathrm{C}), 169.9(\mathrm{C}), 170.2(\mathrm{C})$, 170.4 (C), 170.6 (C); MS m/z (rel int) $722\left(\mathrm{M}^{+}-\mathrm{CH}_{3} \mathrm{O},<1\right), 694$ (1), 634 (1), 331 (66), 169 (100); HRMS m/z calcd for $\mathrm{C}_{32} \mathrm{H}_{36} \mathrm{NO}_{18}$ 722.1932, found 722.1902. Anal. Calcd for $\mathrm{C}_{33} \mathrm{H}_{39} \mathrm{NO}_{19}$ : C, 52.59; H, 5.22; N, 1.86. Found: C, 52.70; H, 5.24; N, 1.99 .

Reductive HAT of Methyl 2,3,4,6-Tetra- $O$-acetyl-r-D-glucopyranosyl-(1f4)-2,3-di- $O$-acetyl-6- $O$-phthalimido- $\beta$-D-glucopyranoside (6). Method A with $n-\mathrm{Bu}_{3} \mathrm{SnH}$. A solution of phthalimide $6(24 \mathrm{mg}, 0.032 \mathrm{mmol})$ in dry benzene $(2.4 \mathrm{~mL})$ containing $n-\mathrm{Bu}_{3} \mathrm{SnH}(9 \mu \mathrm{L}, 0.003 \mathrm{mmol})$ and AIBN (1 mg, 0.003 mmol) was heated at reflux temperature for $1 \mathrm{~h}$. After cooling to room temperature the reaction mixture was concentrated under reduced pressure. The residue was dissolved in $\mathrm{CH}_{3} \mathrm{CN}$, washed with $n$-hexane and the combined more polar extracts were concentrated under reduced pressure. Column chromatography of the crude residue (hexanes-EtOAc, 50:50 f 30:70) afforded methyl 2,3,4,6-tetra- $O$-acetyl-R-D-glucopyranosyl-(1f4)-2,3-di- $O$ acetyl-§-D-glucopyranoside (5) $(1.5 \mathrm{mg}, 0.002 \mathrm{mmol}, 8 \%)$ and methyl 2,3,4,6-tetra- $O$-acetyl- $\$$-L-idopyranosyl-(1f4)-2,3-di- $O$ acetyl-§-D-glucopyranoside (8) (13.5 mg, $0.022 \mathrm{mmol}, 69 \%)$, both as colorless oils. Compound 8: $[R]_{\mathrm{D}}-2.1(c, 0.12)$; IR 3545, 1752 $\mathrm{cm}^{-1}$; ${ }^{1} \mathrm{H}$ NMR $(400 \mathrm{MHz}) \delta_{\mathrm{H}} 2.03(3 \mathrm{H}, \mathrm{s}), 2.04(3 \mathrm{H}, \mathrm{s}), 2.08$ $(3 \mathrm{H}, \mathrm{s}), 2.108(6 \mathrm{H}, \mathrm{s}), 2.111(3 \mathrm{H}, \mathrm{s}), 3.42(1 \mathrm{H}, \mathrm{ddd}, J) \quad 9.5,2.6$, $2.6 \mathrm{~Hz}), 3.49(3 \mathrm{H}, \mathrm{s}), 3.88(1 \mathrm{H}, \mathrm{dd}, J) 12.4,2.6 \mathrm{~Hz}), 3.98(1 \mathrm{H}$, $\mathrm{dd}, J) \quad 9.5,9.5 \mathrm{~Hz}), 4.05(1 \mathrm{H}, \mathrm{dd}, J) \quad 12.7,3.2 \mathrm{~Hz}), 4.14(1 \mathrm{H}$, dd, $J) \quad 10.3 .7 .9 \mathrm{~Hz}), 4.19(1 \mathrm{H}, \mathrm{ddd}, J) \quad 11.1,7.9,1.6 \mathrm{~Hz}), 4.25$ $(1 \mathrm{H}, \mathrm{dd}, J) \quad 10.3,3.2 \mathrm{~Hz}), 4.41(1 \mathrm{H}, \mathrm{d}, J) \quad 7.7 \mathrm{~Hz}), 4.79-4.80$ $(2 \mathrm{H}, \mathrm{m}), 4.84(1 \mathrm{H}, \mathrm{dd}, J) \quad 9.8,7.9 \mathrm{~Hz}), 4.93(1 \mathrm{H}, \mathrm{d}, J) \quad 1.6 \mathrm{~Hz})$, $5.05(1 \mathrm{H}, \mathrm{dd}, J) \quad 2.9,2.9 \mathrm{~Hz}), 5.17(1 \mathrm{H}, \mathrm{dd}, J) \quad 9.8,9.8 \mathrm{~Hz}) ;{ }^{13} \mathrm{C}$ NMR $(100.6 \mathrm{MHz}) \delta_{\mathrm{C}} 20.57\left(2 \times \mathrm{CH}_{3}\right), 20.60\left(\mathrm{CH}_{3}\right), 20.64\left(\mathrm{CH}_{3}\right)$, $20.68\left(\mathrm{CH}_{3}\right), 20.71\left(\mathrm{CH}_{3}\right), 57.1\left(\mathrm{CH}_{3}\right), 61.3\left(\mathrm{CH}_{2}\right), 62.543\left(\mathrm{CH}_{2}\right)$, $65.176(\mathrm{CH}), 66.1(\mathrm{CH}), 67.8(\mathrm{CH}), 71.94(\mathrm{CH}), 71.98(\mathrm{CH}), 74.5$ $(\mathrm{CH}), 74.6(\mathrm{CH}), 76.1(\mathrm{CH}), 98.9(\mathrm{CH}), 101.5(\mathrm{CH}), 167.7(\mathrm{C})$, 169.2 (C), 169.3 (C), 169.4 (C), 169.9 (C), 170.6 (C); MS m/z (rel int) $577\left(\mathrm{M}^{+}-\quad \mathrm{CH}_{3} \mathrm{O}, 4\right), 549(1), 517$ (1), 331 (52), 169 (100); HRMS $m / z$ calcd for $\mathrm{C}_{24} \mathrm{H}_{33} \mathrm{O}_{16} 577.1769$, found 577.1783. Anal. Calcd for $\mathrm{C}_{25} \mathrm{H}_{36} \mathrm{O}_{17}$ : C, 49.34; H, 5.96. Found: C, 49.58; H, 5.74.

Method B with $\boldsymbol{n}$-Bu $3 \mathrm{SnD}$. A solution of phthalimide $\mathbf{6}(28 \mathrm{mg}$, $0.037 \mathrm{mmol})$ in dry benzene $(2.8 \mathrm{~mL})$ containing $n-\mathrm{Bu}_{3} \mathrm{SnD}(10$ $\mu \mathrm{L}, 0.037 \mathrm{mmol})$ and AIBN (1 mg, $0.004 \mathrm{mmol})$ was heated at reflux temperature for $1 \mathrm{~h}$. After this time another portion of $n$ $\mathrm{Bu}_{3} \mathrm{SnD}(10 \mu \mathrm{L}, 0.037 \mathrm{mmol})$ and $\mathrm{AIBN}(1 \mathrm{mg}, 0.004 \mathrm{mmol})$ were added and heating at reflux was continued for an additional $1 \mathrm{~h}$. After cooling to room temperature the reaction mixture was concentrated under reduced pressure. The residue was dissolved in $\mathrm{CH}_{3} \mathrm{CN}$, washed with $n$-hexane and the combined more polar extracts were concentrated under reduced pressure. Column chromatography of the crude residue (hexanes-EtOAc, 50:50 f 30: 70) afforded methyl 2,3,4,6-tetra- $O$-acetyl-R-D-[5-2 $\mathrm{H}]$ glucopyranosyl(1f4)-2,3-di-O-methyl- $\$$-D-glucopyranoside (5-[D]) (4.7 mg, 0.008 mmol, $21 \%,{ }^{1} \mathrm{H} /{ }^{2} \mathrm{H}$ ratio, $8: 2$ by ${ }^{1} \mathrm{H}$ NMR) (contaminated with ca. $10 \%$ of an inseparable, unidentified product) and methyl 2,3,4,6tetra- $O$-acetyl- $\S$-L-(5-2H)idopyranosyl-(1f4)-2,3-di- $O$-acetyl- $\S-D-$ glucopyranoside [8-(D)] (13.4 mg, $0.022 \mathrm{mmol}, 59 \%)$, as colorless oils. Compound (5-[D]): ${ }^{13} \mathrm{C}$ NMR $\delta_{\mathrm{C}} 20.5\left(\mathrm{CH}_{3}\right), 20.56\left(\mathrm{CH}_{3}\right)$, $20.60\left(\mathrm{CH}_{3}\right), 20.63\left(\mathrm{CH}_{3}\right), 20.7\left(\mathrm{CH}_{3}\right), 20.9\left(\mathrm{CH}_{3}\right), 57.2\left(\mathrm{CH}_{3}\right)$, $61.2\left(\mathrm{CH}_{2}\right), 61.794\left(\mathrm{CH}_{2}, \mathrm{C}-6\right.$ '-D), $61.853\left(\mathrm{CH}_{2}\right), 68.139\left(\mathrm{CH}, \mathrm{C}-4^{\prime}-\right.$ D), $68.198(2 \times \mathrm{CH}), 69.4(\mathrm{CH}), 70.2(\mathrm{CH}), 70.4(\mathrm{CH}), 72.2(\mathrm{CH})$, $74.3(\mathrm{CH}), 75.4(\mathrm{CH}), 95.06(\mathrm{CH}), 101.5(\mathrm{CH}), 169.5(\mathrm{C}), 169.6$ (C), $170.0(\mathrm{C}), 170.3(\mathrm{C}), 170.5(\mathrm{C}), 170.7(\mathrm{C}) ; \mathrm{MS} \mathrm{m} / \mathrm{z}$ (rel int) $578\left(\mathrm{M}^{+}-\quad \mathrm{CH}_{3} \mathrm{O},<1\right), 577(<1), 550(<1), 549(<1), 332(12)$, 331 (38), 169 (100); HRMS m/z calcd for $\mathrm{C}_{24} \mathrm{H}_{32}{ }^{2} \mathrm{HO}_{16} 578.1831$, found 578.1829; calcd for $\mathrm{C}_{24} \mathrm{H}_{33} \mathrm{O}_{16} 577.1769$, found 577.1783. Compound 8-(D): ${ }^{1} \mathrm{H}$ NMR (400 MHz) $\delta_{\mathrm{H}} 2.03(3 \mathrm{H}, \mathrm{s}), 2.04(3 \mathrm{H}$, s), $2.08(3 \mathrm{H}, \mathrm{s}), 2.109(6 \mathrm{H}, \mathrm{s}), 2.111(3 \mathrm{H}, \mathrm{s}), 3.42(1 \mathrm{H}, \mathrm{ddd}, J)$ 9.8, 2.9, 2.9 Hz), $3.50(3 \mathrm{H}, \mathrm{s}), 3.88(1 \mathrm{H}$, br d, $J) \quad 12.7 \mathrm{~Hz}), 3.98$ $(1 \mathrm{H}, \mathrm{dd}, J) \quad 9.5,9.5 \mathrm{~Hz}), 4.05(1 \mathrm{H}$, br d, $J) 12.7 \mathrm{~Hz}), 4.14(1 \mathrm{H}$, d, $J) \quad 11.7 \mathrm{~Hz}), 4.25(1 \mathrm{H}, \mathrm{d}, J) \quad 11.7 \mathrm{~Hz}), 4.41(1 \mathrm{H}, \mathrm{d}, J) \quad 8.0$ 
$\mathrm{Hz}), 4.79-4.80(2 \mathrm{H}, \mathrm{m}), 4.85(1 \mathrm{H}, \mathrm{dd}, J)$ 9.5, 7.7 Hz), $4.93(1 \mathrm{H}$, $\mathrm{d}, J) \quad 1.6 \mathrm{~Hz}), 5.06(1 \mathrm{H}, \mathrm{dd}, J) \quad 2.9,2.9 \mathrm{~Hz}), 5.17(1 \mathrm{H}, \mathrm{dd}, J)$ 9.5, 9.5 Hz); ${ }^{13} \mathrm{C}$ NMR $(100.6 \mathrm{MHz}) \delta_{\mathrm{C}} 20.57\left(2 \times \mathrm{CH}_{3}\right), 20.61$ $\left(\mathrm{CH}_{3}\right), 20.65\left(\mathrm{CH}_{3}\right), 20.69\left(\mathrm{CH}_{3}\right), 20.72\left(\mathrm{CH}_{3}\right), 57.1\left(\mathrm{CH}_{3}\right), 61.3$ $\left(\mathrm{CH}_{2}\right), 62.487\left(\mathrm{CH}_{2}\right), 65.127(\mathrm{CH}), 66.1(\mathrm{CH}), 67.8(\mathrm{CH}), 71.9$ $(\mathrm{CH}), 74.5(\mathrm{CH}), 74.6(\mathrm{CH}), 76.1(\mathrm{CH}), 98.9(\mathrm{CH}), 101.5(\mathrm{CH})$, $167.8(\mathrm{CH}), 169.2(\mathrm{CH}), 169.3(\mathrm{C}), 169.4(\mathrm{C}), 169.9(\mathrm{C}), 170.6$ (C); MS m/z (rel int) $578\left(\mathrm{M}^{+}-\mathrm{CH}_{3} \mathrm{O},<1\right), 532(<1), 518$ (1), 332 (31), 170 (100); HRMS $m / z$ calcd for $\mathrm{C}_{24} \mathrm{H}_{32}{ }^{2} \mathrm{HO}_{16} 578.1831$, found 578.1818 .

Methyl 6-Deoxy-2,3,4-tri- $O$-methyl-r-D-talopyranosyl-(1f4)-2,3di- $\boldsymbol{O}$-methyl-6- $\boldsymbol{O}$-phthalimido-r-D-glucopyranoside (10). DEAD (230 $\mu \mathrm{L}, 1.462 \mathrm{mmol}$ ) was added dropwise to a stirred solution of the alcohol 9 (240 mg, $0.585 \mathrm{mmol}), N$-hydroxyphthalimide (238 $\mathrm{mg}, 1.462 \mathrm{mmol})$ and $\mathrm{PPh}_{3}(383 \mathrm{mg}, 1.462 \mathrm{mmol})$ in dry THF $(6.4 \mathrm{~mL})$ and the resulting solution was stirred at $0{ }^{\circ} \mathrm{C}$ for $1.5 \mathrm{~h}$. Then the solvent was removed and the crude was quenched with water and extracted with $\mathrm{Et}_{2} \mathrm{O}$. The combined extracts were dried over $\mathrm{Na}_{2} \mathrm{SO}_{4}$ and concentrated under reduced pressure. The residue obtained was purified by column chromatography $\left(\mathrm{Et}_{2} \mathrm{O}-\mathrm{AcOEt}\right.$, 90:10 f 0:100) to give compound 10 (147 mg, $0.265 \mathrm{mmol}, 45 \%)$ as a foam: $[\mathrm{R}]_{\mathrm{D}}+89.6(c, 0.435)$; IR $1789,1734 \mathrm{~cm}^{-1} ;{ }^{1} \mathrm{H}$ NMR $\left.\left.\delta_{\mathrm{H}} 1.19(3 \mathrm{H}, \mathrm{d}, J) \quad 6.5 \mathrm{~Hz}\right), 3.25(1 \mathrm{H}, \mathrm{dd}, J) \quad 9.5,3.5 \mathrm{~Hz}\right), 3.35$ $(1 \mathrm{H}, \mathrm{m}), 3.42(1 \mathrm{H}, \mathrm{dd}, J) \quad 3.5,3.5 \mathrm{~Hz}), 3.45(3 \mathrm{H}, \mathrm{s}), 3.460(1 \mathrm{H}$, m), $3.463(3 \mathrm{H}, \mathrm{s}), 3.48(3 \mathrm{H}, \mathrm{s}), 3.49(3 \mathrm{H}, \mathrm{s}), 3.52(3 \mathrm{H}, \mathrm{s}), 3.53$ $(1 \mathrm{H}, \mathrm{dd}, J) \quad 9.5,9.5 \mathrm{~Hz}), 3.57(1 \mathrm{H}, \mathrm{dd}, J) \quad 9.0,9.0 \mathrm{~Hz}), 3.60$ $(3 \mathrm{H}, \mathrm{s}), 3.89$ (1H, dddd, $J) \quad 6.5,6.5,6.5,1.5 \mathrm{~Hz}), 3.93(1 \mathrm{H}$, ddd, J) $9.0,7.0,2.0 \mathrm{~Hz}), 4.36(1 \mathrm{H}, \mathrm{dd}, J) \quad 11.5,6.5 \mathrm{~Hz}), 4.42(1 \mathrm{H}$, dd, $J) \quad 11.0,2.0 \mathrm{~Hz}), 4.80(1 \mathrm{H}, \mathrm{d}, J) \quad 3.5 \mathrm{~Hz}), 5.26(1 \mathrm{H}, \mathrm{d}, J)$ $1.5 \mathrm{~Hz}), 7.72-7.76(2 \mathrm{H}, \mathrm{m}), 7.80-7.83(2 \mathrm{H}, \mathrm{m}) ;{ }^{13} \mathrm{C}$ NMR $\delta_{\mathrm{C}}$ $16.5\left(\mathrm{CH}_{3}\right), 55.6\left(\mathrm{CH}_{3}\right), 56.6\left(\mathrm{CH}_{3}\right), 58.7\left(\mathrm{CH}_{3}\right), 59.1\left(\mathrm{CH}_{3}\right), 60.9$ $\left(\mathrm{CH}_{3}\right), 61.4\left(\mathrm{CH}_{3}\right), 67.7(\mathrm{CH}), 69.1(\mathrm{CH}), 76.9(\mathrm{CH}), 77.2(\mathrm{CH})$, $77.3(\mathrm{CH}), 77.8\left(\mathrm{CH}_{2}\right), 78.0(\mathrm{CH}), 81.9(\mathrm{CH}), 82.8(\mathrm{CH}), 97.2(\mathrm{CH})$, $99.7(\mathrm{CH}), 123.5(2 \times \mathrm{CH}), 128.8(2 \times \mathrm{C}), 134.5(2 \times \mathrm{CH}), 163.2$ $(2 \times \mathrm{C}) ; \mathrm{MS} \mathrm{m} / \mathrm{z}(\%) 578\left(\mathrm{M}^{+}+\mathrm{Na}, 100\right), 556\left(\mathrm{M}^{+}+\mathrm{H}, 33\right)$; HRMS $\mathrm{m} / \mathrm{z}$ calcd for $\mathrm{C}_{26} \mathrm{H}_{37} \mathrm{NNaO}_{12}$ 578.2213, found 578.2230. Anal. Calcd for $\mathrm{C}_{26} \mathrm{H}_{37} \mathrm{NO}_{12}$ : C, 56.21; H, 6.71; N, 2.52. Found: C, 56.02; H, 6.92; N, 2.35.

Reductive HAT of Methyl 6-Deoxy-2,3,4-tri- $O$-methyl-r-Dtalopyranosyl-(1f4)-2,3-di- $O$-methyl-6- $O$-phthalimido-r-D-glucopyranoside (10). Method A with $\boldsymbol{n}-\mathrm{Bu}_{3} \mathrm{SnH}$. A solution of phthalimide $10(33 \mathrm{mg}, 0.059 \mathrm{mmol})$ in dry benzene $(4.5 \mathrm{~mL})$ containing $n-\mathrm{Bu}_{3} \mathrm{SnH}(16 \mu \mathrm{L}, 0.059 \mathrm{mmol})$ and AIBN (1 mg, 0.006 $\mathrm{mmol}$ ) was heated at reflux temperature for $1 \mathrm{~h}$. After this time another portion of $n$ - $\mathrm{Bu}_{3} \mathrm{SnH}(16 \mu \mathrm{L}, 0.059 \mathrm{mmol})$ and AIBN (1 $\mathrm{mg}, 0.006 \mathrm{mmol}$ ) were added and heating at reflux was continued for an additional $1 \mathrm{~h}$. After cooling to room temperature the reaction mixture was concentrated under reduced pressure. The residue was dissolved in $\mathrm{CH}_{3} \mathrm{CN}$, washed with $n$-hexane and the combined more polar extracts were concentrated under reduced pressure. Chromatotron chromatography $\left(\mathrm{CHCl}_{3}-\mathrm{MeOH}, 100: 0 \mathrm{f}\right.$ 99:1) gave methyl 6-deoxy-2,3,4-tri- $O$-methyl-§-L-allopyranosyl-(1f4)-2,3-di-O-methylR-D-glucopyranoside (13) (16.5 mg, $0.040 \mathrm{mmol}, 68 \%$ ) and methyl 6-deoxy-2,3,4-tri- $O$-methyl-R-D-talopyranosyl-(1f4)-2,3-di- $O$-methylR-D-glucopyranoside (9) (2 mg, $0.005 \mathrm{mmol}, 8 \%$ ), both as colorless oils. Compound 13: $[\mathrm{R}]_{\mathrm{D}}+87.1(c, 0.56)$; IR $3498 \mathrm{~cm}^{-1} ;{ }^{1} \mathrm{HNMR}$ $\left.(400 \mathrm{MHz}) \delta_{\mathrm{H}} 1.25(3 \mathrm{H}, \mathrm{d}, J) \quad 6.3 \mathrm{~Hz}\right), 2.81(1 \mathrm{H}, \mathrm{dd}, J) \quad 9.6,2.5$ $\mathrm{Hz}), 2.93(1 \mathrm{H}, \mathrm{dd}, J) \quad 7.8,2.5 \mathrm{~Hz}), 3.21(1 \mathrm{H}, \mathrm{dd}, J) \quad 9.6,3.8$ $\mathrm{Hz}), 3.40(3 \mathrm{H}, \mathrm{s}), 3.42(3 \mathrm{H}, \mathrm{s}), 3.517(3 \mathrm{H}, \mathrm{s}), 3.521(3 \mathrm{H}, \mathrm{s}), 3.55$ $(1 \mathrm{H}, \mathrm{ddd}, J) \quad 10.1,2.5,2.5 \mathrm{~Hz}), 3.57(1 \mathrm{H}, \mathrm{dd}, J) \quad 9.3,9.3 \mathrm{~Hz})$, $3.59(3 \mathrm{H}, \mathrm{s}), 3.64(3 \mathrm{H}, \mathrm{s}), 3.65(1 \mathrm{H}, \mathrm{dd}, J) \quad 12.7,2.4 \mathrm{~Hz}), 3.69$ $(1 \mathrm{H}, \mathrm{dd}, J) \quad 10.1,9.1 \mathrm{~Hz}), 3.85(1 \mathrm{H}, \mathrm{dddd}, J) \quad 9.1,6.1,6.1,6.1$ $\mathrm{Hz}), 3.95(1 \mathrm{H}, \mathrm{dd}, J) \quad 12.6,2.5 \mathrm{~Hz}), 3.96(1 \mathrm{H}, \mathrm{dd}, J) \quad 2.5,2.5$ $\mathrm{Hz}), 4.82(1 \mathrm{H}, \mathrm{d}, J) 3.8 \mathrm{~Hz}), 5.09(1 \mathrm{H}, \mathrm{d}, J) 8.1 \mathrm{~Hz}) ;{ }^{13} \mathrm{C} \mathrm{NMR}$ $(100.6 \mathrm{MHz}) \delta_{\mathrm{C}} 17.467\left(\mathrm{CH}_{3}\right), 55.1\left(\mathrm{CH}_{3}\right), 57.4\left(\mathrm{CH}_{3}\right), 59.0\left(\mathrm{CH}_{3}\right)$, $59.3\left(\mathrm{CH}_{3}\right), 61.0\left(\mathrm{CH}_{3}\right), 61.1\left(\mathrm{CH}_{3}\right), 61.7\left(\mathrm{CH}_{2}\right), 68.9(\mathrm{CH}), 70.2$ $(\mathrm{CH}), 75.08(\mathrm{CH}), 75.14(\mathrm{CH}), 81.8(\mathrm{CH}), 82.1(\mathrm{CH}), 83.109(\mathrm{CH})$, $83.5(\mathrm{CH}), 97.9(\mathrm{CH}), 101.063(\mathrm{CH})$; MS m/z $(\%) 379\left(\mathrm{M}^{+}\right.$$\left.\mathrm{CH}_{3} \mathrm{O},<1\right), 346(<1), 307$ (2), 265 (29), 101 (62), 88 (100); HRMS $m / z$ calcd for $\mathrm{C}_{17} \mathrm{H}_{31} \mathrm{O}_{9} 379.1968$, found 379.1975. Anal. Calcd for $\mathrm{C}_{18} \mathrm{H}_{34} \mathrm{O}_{10}$ : C, 52.67; H, 8.35. Found: C, 52.72; H, 8.32.

Method B with $\boldsymbol{n}$-Bu $\mathbf{u}_{3} \mathbf{S n D}$. A solution of phthalimide $\mathbf{1 0}$ (33 $\mathrm{mg}, 0.059 \mathrm{mmol})$ in dry benzene $(4.5 \mathrm{~mL})$ containing $n-\mathrm{Bu}_{3} \mathrm{SnD}$ $(16 \mu \mathrm{L}, 0.059 \mathrm{mmol})$ and AIBN $(1 \mathrm{mg}, 0.006 \mathrm{mmol})$ was heated at reflux temperature for $1 \mathrm{~h}$. After this time another portion of $n$ $\mathrm{Bu}_{3} \mathrm{SnD}(16 \mu \mathrm{L}, 0.059 \mathrm{mmol})$ and $\mathrm{AIBN}(1 \mathrm{mg}, 0.006 \mathrm{mmol})$ were added and heating at reflux was continued for an additional $1 \mathrm{~h}$. After cooling to room temperature the reaction mixture was concentrated under reduced pressure. The residue was dissolved in $\mathrm{CH}_{3} \mathrm{CN}$, washed with $n$-hexane and the combined more polar extracts were concentrated under reduced pressure. Chromatotron chromatography $\left(\mathrm{CHCl}_{3}-\mathrm{MeOH}, 100: 0\right.$ f 99:1) gave methyl 2,3,4tri- $O$-acetyl-6-deoxy- $\S-\mathrm{L}-(5-2 \mathrm{H})$ allopyranosyl-(1f4)-2,3-di- $O$-methyl-R-D-glucopyranoside [13-(D)] (13.6 mg, $0.033 \mathrm{mmol}, 56 \%)$ and methyl 2,3,4-tri- $O$-acetyl-6-deoxy-R-D-[5-2H]talopyranosyl-(1f4)2,3-di- $O$-methyl-R-D-glucopyranoside (9-[D]) $(1.5 \mathrm{mg}, 0.004 \mathrm{mmol}$, $6 \%,{ }^{1} \mathrm{H} /{ }^{2} \mathrm{H}$ ratio, 3:7), both as colorless oils. Compound 13-(D): ${ }^{1} \mathrm{H}$ NMR $\left.(400 \mathrm{MHz}) \delta_{\mathrm{H}} 1.24(3 \mathrm{H}, \mathrm{s}), 2.81(1 \mathrm{H}, \mathrm{d}, J) 2.4 \mathrm{~Hz}\right)$, $2.93(1 \mathrm{H}, \mathrm{dd}, J) \quad 7.9,2.4 \mathrm{~Hz}), 3.21(1 \mathrm{H}, \mathrm{dd}, J) \quad 9.5,3.7 \mathrm{~Hz})$, $3.39(3 \mathrm{H}, \mathrm{s}), 3.41(3 \mathrm{H}, \mathrm{s}), 3.51(3 \mathrm{H}, \mathrm{s}), 3.52(3 \mathrm{H}, \mathrm{s}), 3.55(1 \mathrm{H}$, ddd, $J$ ) 9.8, 2.6, $2.6 \mathrm{~Hz}), 3.57(1 \mathrm{H}, \mathrm{dd}, J) \quad 9.3,9.3 \mathrm{~Hz}), 3.59$ $(3 \mathrm{H}, \mathrm{s}), 3.64(3 \mathrm{H}, \mathrm{s}), 3.66(1 \mathrm{H}, \mathrm{m}), 3.69(1 \mathrm{H}, \mathrm{dd}, J) \quad 10.1,9.0$ $\mathrm{Hz}), 3.94(1 \mathrm{H}, \mathrm{dd}, J) \quad 12.7,2.6 \mathrm{~Hz}), 3.96(1 \mathrm{H}, \mathrm{dd}, J) \quad 2.4,2.4$ $\mathrm{Hz}), 4.81(1 \mathrm{H}, \mathrm{d}, J) 3.7 \mathrm{~Hz}), 5.09(1 \mathrm{H}, \mathrm{d}, J) 8.0 \mathrm{~Hz}) ;{ }^{13} \mathrm{C} \mathrm{NMR}$ $(100.6 \mathrm{MHz}) \delta_{\mathrm{C}} 17.328\left(\mathrm{CH}_{3}\right), 55.1\left(\mathrm{CH}_{3}\right), 57.4\left(\mathrm{CH}_{3}\right), 59.0\left(\mathrm{CH}_{3}\right)$, $59.3\left(\mathrm{CH}_{3}\right), 60.9\left(\mathrm{CH}_{3}\right), 61.1\left(\mathrm{CH}_{3}\right), 61.7\left(\mathrm{CH}_{2}\right), 70.2(\mathrm{CH}), 75.06$ $(\mathrm{CH}), 75.13(\mathrm{CH}), 81.8(\mathrm{CH}), 82.1(\mathrm{CH}), 83.002(\mathrm{CH}), 83.5(\mathrm{CH})$, $97.9(\mathrm{CH}), 101.031(\mathrm{CH})$; MS m/z $(\%) 380\left(\mathrm{M}^{+}-\mathrm{CH}_{3} \mathrm{O},<1\right)$, 307 (2), 265 (24), 101 (50), 88 (100); HRMS m/z calcd for $\mathrm{C}_{17} \mathrm{H}_{30}{ }^{2} \mathrm{HO}_{9} 380.2031$ found 380.2045. Compound 9-[D]: ${ }^{1} \mathrm{H}$ NMR $\left.\delta_{\mathrm{H}} 1.33(3 \mathrm{H}, \mathrm{s}), 3.24(1 \mathrm{H}, \mathrm{dd}, J) \quad 9.0,3.4 \mathrm{~Hz}\right), 3.36(1 \mathrm{H}, \mathrm{dd}, J)$ 2.9, $0.8 \mathrm{~Hz}), 3.42(3 \mathrm{H}, \mathrm{s}), 3.44-3.47(2 \mathrm{H}, \mathrm{m}), 3.49(3 \mathrm{H}, \mathrm{s}), 3.50$ $(3 \mathrm{H}, \mathrm{s}), 3.51(3 \mathrm{H}, \mathrm{s}), 3.52(1 \mathrm{H}, \mathrm{m}), 3.55(3 \mathrm{H}, \mathrm{s}), 3.56-3.60(2 \mathrm{H}$, m), $3.62(3 \mathrm{H}, \mathrm{s}), 3.73-3.81(2 \mathrm{H}, \mathrm{m}), 4.83(1 \mathrm{H}, \mathrm{d}, J) 3.7 \mathrm{~Hz})$, $5.29(1 \mathrm{H}, \mathrm{d}, J) \quad 2.1 \mathrm{~Hz}) ;{ }^{13} \mathrm{C} \mathrm{NMR}(100.6 \mathrm{MHz}) \delta_{\mathrm{C}} 16.370\left(\mathrm{CH}_{3}\right)$, $16.496\left(\mathrm{CH}_{3}\right), 55.2\left(\mathrm{CH}_{3}\right), 57.1\left(\mathrm{CH}_{3}\right), 58.8\left(\mathrm{CH}_{3}\right), 59.2\left(\mathrm{CH}_{3}\right)$, $61.0\left(\mathrm{CH}_{3}\right), 61.1\left(\mathrm{CH}_{3}\right), 62.1\left(\mathrm{CH}_{2}\right), 68.5(\mathrm{CH}), 70.3(\mathrm{CH}), 76.8$ $(\mathrm{CH}), 77.5(\mathrm{CH}), 77.6(\mathrm{CH}), 78.011(\mathrm{CH}), 78.074(\mathrm{CH}), 82.4(\mathrm{CH})$, $83.2(\mathrm{CH}), 97.4(\mathrm{CH}), 100.051(\mathrm{CH}), 100.079(\mathrm{CH})$; MS (FAB) $\mathrm{m} / \mathrm{z}(\%) 434\left(\mathrm{M}^{+}+\mathrm{Na}, 100\right), 433(28)$; HRMS $\mathrm{m} / \mathrm{z}$ calcd for $\mathrm{C}_{1} \mathrm{H}{ }_{3}^{2} \mathrm{H} \mathrm{NaO} 434.2112$, found 434.2133; calcd for $\mathrm{C} \mathrm{H} \mathrm{NaO}_{34}$ 433.2050, found 433.2050.

Methyl 2,3,4-Tri- $O$-acetyl-6-deoxy-r-D-talopyranosyl-(1f4)-2,3di- $\boldsymbol{O}$-methyl-6- $\boldsymbol{O}$-phthalimido-r-D-glucopyranoside (12). DEAD (120 $\mu \mathrm{L}, 0.760 \mathrm{mmol}$ ) was added dropwise to a stirred solution of the alcohol 11 (150 mg, $0.304 \mathrm{mmol}), N$-hydroxyphthalimide (124 $\mathrm{mg}, 0.760 \mathrm{mmol})$ and $\mathrm{PPh}_{3}(199 \mathrm{mg}, 0.760 \mathrm{mmol})$ in dry THF $(3.3 \mathrm{~mL})$ and the resulting solution was stirred at $0{ }^{\circ} \mathrm{C}$ for $1 \mathrm{~h}$. Then the solvent was removed and the crude was quenched with water and extracted with $\mathrm{Et}_{2} \mathrm{O}$. The combined extracts were dried over $\mathrm{Na}_{2} \mathrm{SO}_{4}$ and concentrated under reduced pressure. The residue obtained was purified by column chromatography (hexanes- $\mathrm{Et}_{2} \mathrm{O}$, 50:50 f 0:100) to give compound 12 (187 mg, $0.293 \mathrm{mmol}, 96 \%)$ as a colorless oil: $[\mathrm{R}]_{\mathrm{D}}+99.2(c, 0.36)$; IR $1789,1738 \mathrm{~cm}^{-1} ;{ }^{1} \mathrm{H}$ NMR $\left.(400 \mathrm{MHz}) \delta_{\mathrm{H}} 1.16(3 \mathrm{H}, \mathrm{d}, J) \quad 6.3 \mathrm{~Hz}\right), 1.98(3 \mathrm{H}, \mathrm{s}), 2.12$ $(3 \mathrm{H}, \mathrm{s}), 2.14(3 \mathrm{H}, \mathrm{s}), 3.26(1 \mathrm{H}, \mathrm{dd}, J) \quad 9.5,3.4 \mathrm{~Hz}), 3.46(3 \mathrm{H}, \mathrm{s})$, $3.49(3 \mathrm{H}, \mathrm{s}), 3.58(1 \mathrm{H}, \mathrm{dd}, J) 9.5,8.7 \mathrm{~Hz}), 3.59(3 \mathrm{H}, \mathrm{s}), 3.76$ $(1 \mathrm{H}, \mathrm{dd}, J) \quad 10.1,8.7 \mathrm{~Hz}), 3.92(1 \mathrm{H}, \mathrm{ddd}, J) \quad 10.1,3.4,3.4 \mathrm{~Hz})$, $4.26(1 \mathrm{H}$, dddd, $J) \quad 6.3,6.3,6.3,1.6 \mathrm{~Hz}), 4.41(2 \mathrm{H}, \mathrm{d}, J) 3.4$ $\mathrm{Hz}), 4.78(1 \mathrm{H}, \mathrm{d}, J) \quad 3.4 \mathrm{~Hz}), 5.17(1 \mathrm{H}, \mathrm{ddd}, J) \quad 3.4,1.6,1.6$ $\mathrm{Hz}), 5.20(1 \mathrm{H}, \mathrm{ddd}, J)$ 3.7, 1.6, $1.6 \mathrm{~Hz}), 5.25(1 \mathrm{H}, \mathrm{d}, J) 1.6$ $\mathrm{Hz}), 5.26(1 \mathrm{H}, \mathrm{dd}, J)$ 3.7, $3.7 \mathrm{~Hz}), 7.75(2 \mathrm{H}, \mathrm{m}), 7.83(2 \mathrm{H}, \mathrm{m})$; ${ }^{13} \mathrm{C} \mathrm{NMR}(100.6 \mathrm{MHz}) \delta_{\mathrm{C}} 16.1\left(\mathrm{CH}_{3}\right), 20.6\left(\mathrm{CH}_{3}\right), 20.7\left(\mathrm{CH}_{3}\right)$, $20.9\left(\mathrm{CH}_{3}\right), 55.7\left(\mathrm{CH}_{3}\right), 58.8\left(\mathrm{CH}_{3}\right), 61.0\left(\mathrm{CH}_{3}\right), 65.8(\mathrm{CH}), 65.9$ $(\mathrm{CH}), 67.3(\mathrm{CH}), 68.9(\mathrm{CH}), 69.0(\mathrm{CH}), 76.8(\mathrm{CH}), 77.1\left(\mathrm{CH}_{2}\right)$, $81.9(\mathrm{CH}), 82.7(\mathrm{CH}), 97.4(\mathrm{CH}), 100.4(\mathrm{CH}), 123.6(2 \times \mathrm{CH})$, $128.9(2 \times \mathrm{C}), 134.5(2 \times \mathrm{CH}), 163.2(2 \times \mathrm{C}), 169.6(\mathrm{C}), 169.8$ (C), $170.6(\mathrm{C}) ; \mathrm{MS} \mathrm{m} / z$ (\%) $579\left(\mathrm{M}^{+}-\mathrm{AcOH},<1\right), 435$ (1), 273 
(96), 88 (100); HRMS m/z calcd for $\mathrm{C}_{27} \mathrm{H}_{33} \mathrm{NO}_{13}$ 579.1952, found 579.1948. Anal. Calcd for $\mathrm{C}_{29} \mathrm{H}_{37} \mathrm{NO}_{15}: \mathrm{C}, 54.46 ; \mathrm{H}, 5.83 ; \mathrm{N}, 2.19$. Found: C, 54.13; H, 6.22; N, 2.30.

Reductive HAT of Methyl 2,3,4-Tri- $O$-acetyl-6-deoxy-r-Dtalopyranosyl-(1f4)-2,3-di- $O$-methyl-6- $O$-phthalimido-r-D-glucopyranoside (12). Method A with $\boldsymbol{n}$ - $\mathrm{Bu}_{3} \mathrm{SnH}$. A solution of phthalimide $12(41 \mathrm{mg}, 0.064 \mathrm{mmol})$ in dry benzene $(4.8 \mathrm{~mL})$ containing $n-\mathrm{Bu}_{3} \mathrm{SnH}(17 \mu \mathrm{L}, 0.064 \mathrm{mmol})$ and AIBN (1 mg, 0.006 mmol) was heated at reflux temperature for $2 \mathrm{~h}$. After this time another portion of $n-\mathrm{Bu}_{3} \mathrm{SnH}(17 \mu \mathrm{L}, 0.064 \mathrm{mmol})$ and AIBN (1 $\mathrm{mg}, 0.006 \mathrm{mmol}$ ) were added and heating at reflux was continued for an additional $1 \mathrm{~h}$. After cooling to room temperature the reaction mixture was concentrated under reduced pressure. The residue was dissolved in $\mathrm{CH}_{3} \mathrm{CN}$, washed with $n$-hexane and the combined more polar extracts were concentrated under reduced pressure. Chromatotron chromatography (hexanes-EtOAc, 60:40 f 40:60) gave methyl (5R)-5,6-anhydro-2,3-di-O-acetyl-4,6-dideoxy-R-L-erythrohexos-5-ulopyranosyl-(1f4)-2,3-di-O-methyl-R-D-glucopyranoside (15) (6 mg, $0.014 \mathrm{mmol}, 22 \%)$, methyl 2,3,4-tri- $O$-acetyl-6deoxy- $\S$ - L-allopyranosyl-(1 f4 )-2,3-di - $O$-methyl- R-Dglucopyranoside (14) (5.5 mg, $0.011 \mathrm{mmol}, 17 \%$ ), and methyl 2,3,4tri- $O$-acetyl-6-deoxy-R-D-talopyranosyl-(1f4)-2,3-di- $O$-methyl-RD-glucopyranoside (11) (12 mg, $0.024 \mathrm{mmol}, 38 \%$ ), all as colorless oils. Compound 15: [R $]_{\mathrm{D}}+77.7(c, 0.26)$; IR $1748 \mathrm{~cm}^{-1}$; ${ }^{1} \mathrm{HNMR}$ $\left.\delta_{\mathrm{H}} 1.40(3 \mathrm{H}, \mathrm{s}), 1.98(1 \mathrm{H}, \mathrm{dd}, J) \quad 12.6,12.6 \mathrm{~Hz}\right), 2.01(3 \mathrm{H}, \mathrm{s})$, $2.06(1 \mathrm{H}, \mathrm{dd}, J) \quad 12.6,4.8 \mathrm{~Hz}), 2.13(3 \mathrm{H}, \mathrm{s}), 3.14(1 \mathrm{H}, \mathrm{dd}, J)$ 9.2, $3.6 \mathrm{~Hz}), 3.40(3 \mathrm{H}, \mathrm{s}), 3.49(1 \mathrm{H}, \mathrm{dd}, J) \quad 9.0,9.0 \mathrm{~Hz}), 3.51$ $(3 \mathrm{H}, \mathrm{s}), 3.56(1 \mathrm{H}, \mathrm{dd}, J)$ 9.0, $9.0 \mathrm{~Hz}), 3.58(3 \mathrm{H}, \mathrm{s}), 3.61-3.66$ $(2 \mathrm{H}, \mathrm{m}), 3.83(1 \mathrm{H}, \mathrm{dd}, J) \quad 12.3,11.2 \mathrm{~Hz}), 4.75(1 \mathrm{H}, \mathrm{d}, J) \quad 3.6$ $\mathrm{Hz}), 4.96(1 \mathrm{H}, \mathrm{d}, J) 1.7 \mathrm{~Hz}), 5.21(1 \mathrm{H}, \mathrm{m}), 5.52(1 \mathrm{H}, \mathrm{ddd}, J)$ 12.0, 5.0, 3.1 Hz); ${ }^{13} \mathrm{C}$ NMR $\delta_{\mathrm{C}} 20.88\left(\mathrm{CH}_{3}\right), 20.94\left(\mathrm{CH}_{3}\right), 25.4$ $\left(\mathrm{CH}_{3}\right), 35.375\left(\mathrm{CH}_{2}\right), 55.1\left(\mathrm{CH}_{3}\right), 59.1\left(\mathrm{CH}_{3}\right), 61.4\left(\mathrm{CH}_{3}\right), 64.0$ $\left(\mathrm{CH}_{2}\right), 64.355(\mathrm{CH}), 66.1(\mathrm{CH}), 67.3(\mathrm{CH}), 79.6(\mathrm{CH}), 81.1(\mathrm{CH})$, $81.5(\mathrm{CH}), 97.6(\mathrm{CH}), 98.4(\mathrm{CH}), 100.626(\mathrm{C}), 169.8(\mathrm{C}), 170.0$ (C); MS m/z (\%) 434 (M+, 2), 403 (3), 374 (5), 273 (29), 101(50), 88 (100); HRMS m/z calcd for $\mathrm{C}_{19} \mathrm{H}_{30} \mathrm{O}_{11}$ 434.1788, found 434.1780. Anal. Calcd for $\mathrm{C}_{19} \mathrm{H}_{30} \mathrm{O}_{11}$ : C, 52.53; H, 6.96. Found: C, 52.81; H, 6.62. Compound 14: $[\mathrm{R}]_{\mathrm{D}}+60.3(c, 0.36)$; IR 3520, $1752 \mathrm{~cm}^{-1}$; ${ }^{1} \mathrm{H}$ NMR $\left.(400 \mathrm{MHz}) \delta_{\mathrm{H}} 1.23(3 \mathrm{H}, \mathrm{d}, J) 6.1 \mathrm{~Hz}\right)$, $2.014(3 \mathrm{H}, \mathrm{s}), 2.015(3 \mathrm{H}, \mathrm{s}), 2.13(3 \mathrm{H}, \mathrm{s}), 3.22(1 \mathrm{H}, \mathrm{dd}, J) \quad 9.5$, $3.7 \mathrm{~Hz}), 3.40(3 \mathrm{H}, \mathrm{s}), 3.52(3 \mathrm{H}, \mathrm{s}), 3.54(1 \mathrm{H}, \mathrm{dd}, J) \quad 9.5,9.5 \mathrm{~Hz})$, $3.55(1 \mathrm{H}, \mathrm{m}), 3.62(3 \mathrm{H}, \mathrm{s}), 3.68(1 \mathrm{H}$, br dd, $J) \quad 12.4,1.9 \mathrm{~Hz})$, $3.72(1 \mathrm{H}, \mathrm{dd}, J) \quad 10.1,9.3 \mathrm{~Hz}), 3.94(1 \mathrm{H}, \mathrm{br} \mathrm{dd}, J) \quad 12.5,2.9$ $\mathrm{Hz}), 4.00$ (1H, dddd, $J$ ) 9.8, 6.1, 6.1, $6.1 \mathrm{~Hz}), 4.70(1 \mathrm{H}, \mathrm{dd}, J)$ 10.1, $2.9 \mathrm{~Hz}), 4.81(1 \mathrm{H}, \mathrm{dd}, J) \quad 8.5,2.9 \mathrm{~Hz}), 4.84(1 \mathrm{H}, \mathrm{d}, J) 3.7$ $\mathrm{Hz}), 5.22(1 \mathrm{H}, \mathrm{d}, J) \quad 8.5 \mathrm{~Hz}), 5.60(1 \mathrm{H}, \mathrm{dd}, J) \quad 2.9,2.9 \mathrm{~Hz}) ;{ }^{13} \mathrm{C}$ NMR $(100.6 \mathrm{MHz}) \delta_{\mathrm{C}} 17.157\left(\mathrm{CH}_{3}\right), 20.57\left(2 \times \mathrm{CH}_{3}\right), 20.63\left(\mathrm{CH}_{3}\right)$, $55.2\left(\mathrm{CH}_{3}\right), 59.0\left(\mathrm{CH}_{3}\right), 61.0\left(\mathrm{CH}_{3}\right), 61.3\left(\mathrm{CH}_{2}\right), 68.5(\mathrm{CH}), 68.6$ $(\mathrm{CH}), 69.7(\mathrm{CH}), 70.0(\mathrm{CH}), 71.062(\mathrm{CH}), 74.5(\mathrm{CH}), 82.3(\mathrm{CH})$, $83.4(\mathrm{CH}), 97.6(\mathrm{CH}), 98.8(\mathrm{CH}), 169.0(\mathrm{C}), 169.3(\mathrm{C}), 169.8(\mathrm{C})$; MS m/z (\%) 434 (M+ - AcOH, 1), 390 (1), 375 (1), 273 (18), 105 (100), 88 (80); HRMS $m / z$ calcd for $\mathrm{C}_{19} \mathrm{H}_{30} \mathrm{O}_{11} 434.1788$, found 434.1795. Anal. Calcd for $\mathrm{C}_{21} \mathrm{H}_{34} \mathrm{O}_{13}$ : C, 51.01; H, 6.93. Found: C, 51.06; H, 6.94.

Method B with $\boldsymbol{n}$-Bu $\mathbf{B} \mathbf{S n D}$. A solution of phthalimide 12 (44 $\mathrm{mg}, 0.069 \mathrm{mmol})$ in dry benzene $(5.2 \mathrm{~mL})$ containing $n-\mathrm{Bu}_{3} \mathrm{SnD}$ (19 $\mu \mathrm{L}, 0.069 \mathrm{mmol})$ and AIBN (1 mg, $0.007 \mathrm{mmol})$ was heated at reflux temperature for $2 \mathrm{~h}$. After this time another portion of $n$ $\mathrm{Bu}_{3} \mathrm{SnD}(19 \mu \mathrm{L}, 0.069 \mathrm{mmol})$ and $\operatorname{AIBN}(1 \mathrm{mg}, 0.007 \mathrm{mmol})$ were added and heating at reflux was continued for an additional $1 \mathrm{~h}$. After cooling to room temperature the reaction mixture was concentrated under reduced pressure. The residue was dissolved in $\mathrm{CH}_{3} \mathrm{CN}$, washed with $n$-hexane and the combined more polar extracts were concentrated under reduced pressure. Chromatotron chromatography (hexanes-EtOAc, 60:40 f 40:60) gave methyl 5,6-anhydro-2,3-di- $O$-acetyl-4,6-dideoxy- $\S$-L-[4-2 $\mathrm{H}]$ ribo-hexos-5ulopyranosyl-(1f4)-2,3-di- $O$-methyl-R-D-glucopyranoside (16) (13 $\mathrm{mg}, 0.014 \mathrm{mmol}, 43 \%,{ }^{1} \mathrm{H} /{ }^{2} \mathrm{H}$ ratio, $\left.1: 6\right)$, methyl 2,3,4-tri- $O$-acetyl6-deoxy-\$-L-(5-2H)allopyranosyl-(1f4)-2,3-di- $O$-methyl-R-D-glu- copyranoside [14-(D)] (4.2 mg, $0.008 \mathrm{mmol}, 12 \%)$, and methyl 2,3,4-tri- $O$-acetyl-6-deoxy-R-D-(5- $\left.{ }^{2} \mathrm{H}\right)$ talopyranosyl-(1f4)-2,3-di$O$-methyl-R-D-glucopyranoside [11-(D)] $(9.3 \mathrm{mg}, 0.019 \mathrm{mmol}$, 27\%), all as colorless oils. Compound 16: ${ }^{1} \mathrm{H}$ NMR (Only the deuterated compound is described) $\delta_{\mathrm{H}} 1.40(3 \mathrm{H}, \mathrm{s}), 2.01(3 \mathrm{H}, \mathrm{s})$, $2.035(1 \mathrm{H}$, br d, $J) \quad 5.0 \mathrm{~Hz}), 2.13(3 \mathrm{H}, \mathrm{s}), 3.14(1 \mathrm{H}, \mathrm{dd}, J) \quad 9.2$, $3.6 \mathrm{~Hz}), 3.40(3 \mathrm{H}, \mathrm{s}), 3.49(1 \mathrm{H}, \mathrm{dd}, J) \quad 9.0,9.0 \mathrm{~Hz}), 3.51(3 \mathrm{H}, \mathrm{s})$, $3.55(1 \mathrm{H}, \mathrm{dd}, J) \quad 9.0,9.0 \mathrm{~Hz}), 3.58(3 \mathrm{H}, \mathrm{s}), 3.62-3.66(2 \mathrm{H}, \mathrm{m})$, $3.83(1 \mathrm{H}, \mathrm{dd}, J) \quad 12.3,11.2 \mathrm{~Hz}), 4.75(1 \mathrm{H}, \mathrm{d}, J) \quad 3.6 \mathrm{~Hz}), 4.96$ $(1 \mathrm{H}, \mathrm{d}, J) 1.7 \mathrm{~Hz}), 5.21(1 \mathrm{H}, \mathrm{m}), 5.51(1 \mathrm{H}, \mathrm{dd}, J) \quad 4.8,3.1 \mathrm{~Hz})$; ${ }^{13} \mathrm{C}$ NMR $\delta_{\mathrm{C}} 20.88\left(\mathrm{CH}_{3}\right), 20.94\left(\mathrm{CH}_{3}\right), 25.4\left(\mathrm{CH}_{3}\right), 35.042(\mathrm{CH}$, t, $\left.\left.J_{\mathrm{CD}}\right) \quad 19.6 \mathrm{~Hz}\right), 35.372\left(\mathrm{CH}_{2}\right), 55.1\left(\mathrm{CH}_{3}\right), 59.1\left(\mathrm{CH}_{3}\right), 61.4$ $\left(\mathrm{CH}_{3}\right), 64.0\left(\mathrm{CH}_{2}\right), 64.306(\mathrm{CH}), 64.352(\mathrm{CH}), 66.1(\mathrm{CH}), 67.3$ $(\mathrm{CH}), 79.6(\mathrm{CH}), 81.1(\mathrm{CH}), 81.5(\mathrm{CH}), 97.6(\mathrm{CH}), 98.4(\mathrm{CH})$, 100.598 (C), 169.8 (C), 170.0 (C); MS m/z (\%) $435\left(\mathrm{M}^{+}, 1\right), 404$ (1), 375 (1), 274 (13), 101 (46), 88 (100); HRMS m/z calcd for $\mathrm{C}_{19} \mathrm{H}_{29}{ }^{2} \mathrm{HO}_{11}$ 435.1851, found 435.1842. Compound 14-(D): ${ }^{1} \mathrm{H}$ NMR $\delta_{\mathrm{H}} 1.22(3 \mathrm{H}, \mathrm{s}), 2.01(6 \mathrm{H}, \mathrm{s}), 2.13(3 \mathrm{H}, \mathrm{s}), 2.71(1 \mathrm{H}, \mathrm{br} \mathrm{s})$, $3.22(1 \mathrm{H}, \mathrm{dd}, J)$ 9.5, $3.8 \mathrm{~Hz}), 3.40(3 \mathrm{H}, \mathrm{s}), 3.52(3 \mathrm{H}, \mathrm{s}), 3.54$ $(1 \mathrm{H}, \mathrm{dd}, J) \quad 9.5,9.5 \mathrm{~Hz}), 3.55(1 \mathrm{H}, \mathrm{m}), 3.62(3 \mathrm{H}, \mathrm{s}), 3.68(1 \mathrm{H}$, m), $3.72(1 \mathrm{H}, \mathrm{dd}, J) \quad 9.2,9.2 \mathrm{~Hz}), 3.94(1 \mathrm{H}$, br d, $J) \quad 12.9 \mathrm{~Hz})$, $4.69(1 \mathrm{H}, \mathrm{d}, J) 2.8 \mathrm{~Hz}), 4.81(1 \mathrm{H}, \mathrm{dd}, J) \quad 8.1,2.8 \mathrm{~Hz}), 4.84(1 \mathrm{H}$, d, $J) \quad 3.6 \mathrm{~Hz}), 5.22(1 \mathrm{H}, \mathrm{d}, J) \quad 8.1 \mathrm{~Hz}), 5.60(1 \mathrm{H}, \mathrm{dd}, J) \quad 2.8$, $2.8 \mathrm{~Hz}) ;{ }^{13} \mathrm{C} \mathrm{NMR} \delta_{\mathrm{C}} 17.022\left(\mathrm{CH}_{3}\right), 20.57\left(2 \times \mathrm{CH}_{3}\right), 20.63\left(\mathrm{CH}_{3}\right)$, $55.2\left(\mathrm{CH}_{3}\right), 59.0\left(\mathrm{CH}_{3}\right), 61.0\left(\mathrm{CH}_{3}\right), 61.3\left(\mathrm{CH}_{2}\right), 68.6(\mathrm{CH}), 69.7$ $(\mathrm{CH}), 70.0(\mathrm{CH}), 70.985(\mathrm{CH}), 74.5(\mathrm{CH}), 82.3(\mathrm{CH}), 83.4(\mathrm{CH})$, $97.6(\mathrm{CH}), 98.8(\mathrm{CH}), 169.0(\mathrm{C}), 169.3(\mathrm{C}), 169.8(\mathrm{C}) ; \mathrm{MS} \mathrm{m} / \mathrm{z}$ (\%) $435\left(\mathrm{M}^{+}\right.$- $\left.\mathrm{AcOH}, 7\right), 274$ (98), 101 (24), 88 (100); HRMS $\mathrm{m} / \mathrm{z}$ calcd for $\mathrm{C}_{19} \mathrm{H}_{29}{ }^{2} \mathrm{HO}_{11}$ 435.1851, found 435.1843. Compound 11-(D): ${ }^{1} \mathrm{H}$ NMR $(400 \mathrm{MHz}) \delta_{\mathrm{H}} 1.22(3 \mathrm{H}, \mathrm{s}), 2.00(3 \mathrm{H}, \mathrm{s}), 2.13$ $(3 \mathrm{H}, \mathrm{s}), 2.15(3 \mathrm{H}, \mathrm{s}), 3.21(1 \mathrm{H}, \mathrm{dd}, J) \quad 9.3,3.4 \mathrm{~Hz}), 3.43(3 \mathrm{H}, \mathrm{s})$, $3.50(3 \mathrm{H}, \mathrm{s}), 3.56(1 \mathrm{H}, \mathrm{dd}, J) 9.3,9.3 \mathrm{~Hz}), 3.60(3 \mathrm{H}, \mathrm{s}), 3.61-3.64$ $(2 \mathrm{H}, \mathrm{m}, 4-\mathrm{H}), 3.75(1 \mathrm{H}$, br dd, $J) \quad 12.2,2.9 \mathrm{~Hz}), 3.81(1 \mathrm{H}, \mathrm{dd}, J$ ) $12.2,1.1 \mathrm{~Hz}), 4.82(1 \mathrm{H}, \mathrm{d}, J) 3.4 \mathrm{~Hz}), 5.15(1 \mathrm{H}, \mathrm{m}), 5.19(1 \mathrm{H}$, ddd, $J$ ) $4.0,1.6,1.1 \mathrm{~Hz}), 5.25(1 \mathrm{H}, \mathrm{dd}, J) \quad 3.7,3.7 \mathrm{~Hz}), 5.25$ $(1 \mathrm{H}, \mathrm{m}) ;{ }^{13} \mathrm{C} \mathrm{NMR}(100.6 \mathrm{MHz}) \delta_{\mathrm{C}} 15.996\left(\mathrm{CH}_{3}\right), 20.59\left(\mathrm{CH}_{3}\right)$, $20.63\left(\mathrm{CH}_{3}\right), 20.8\left(\mathrm{CH}_{3}\right), 55.2\left(\mathrm{CH}_{3}\right), 58.8\left(\mathrm{CH}_{3}\right), 61.2\left(\mathrm{CH}_{3}\right), 61.8$ $\left(\mathrm{CH}_{2}\right), 65.9(\mathrm{CH}), 67.3(\mathrm{CH}), 68.715(\mathrm{CH}), 70.0(\mathrm{CH}), 76.5(\mathrm{CH})$, $82.4(\mathrm{CH}), 83.0(\mathrm{CH}), 97.4(\mathrm{CH}), 100.4(\mathrm{CH}), 169.7(\mathrm{C}), 169.8$ (C), 170.5 (C); MS m/z (\%) $435\left(\mathrm{M}^{+}\right.$- $\left.\mathrm{AcOH}, 2\right), 274$ (61), 101 (22), 88 (100); HRMS m/z calcd for $\mathrm{C}_{19} \mathrm{H}_{29}{ }^{2} \mathrm{HO}_{11} 435.1851$, found 435.1849 .

Methyl 2,3,4,6-Tetra- $O$-methyl-r-D-mannopyranosyl-(1f4)-2,3di- $\boldsymbol{O}$-methyl-6- $\boldsymbol{O}$-phthalimido-r-D-glucopyranoside (18). DEAD (330 $\mu \mathrm{L}, 2.09 \mathrm{mmol}$ ) was added dropwise to a stirred solution of the alcohol 17 (368 mg, $0.836 \mathrm{mmol}), N$-hydroxyphthalimide (272 $\mathrm{mg}, 1.67 \mathrm{mmol})$ and $\mathrm{PPh}_{3}(548 \mathrm{mg}, 2.09 \mathrm{mmol})$ in dry THF $(9.2$ $\mathrm{mL}$ ) and the resulting solution was stirred at $0{ }^{\circ} \mathrm{C}$ for $1 \mathrm{~h}$. Then the solvent was removed and the crude was quenched with water and extracted with $\mathrm{CHCl}_{3}$. The combined extracts were dried over $\mathrm{Na}_{2} \mathrm{SO}_{4}$ and concentrated under reduced pressure. The residue obtained was purified by column chromatography (hexanes-EtOAc, 30:70) to give compound 18 (224 $\mathrm{mg}, 0.383 \mathrm{mmol}, 46 \%)$ as a white foam: $[R]_{\mathrm{D}}+76.0(c, 0.213)$; IR 1790, $1734 \mathrm{~cm}^{-1} ;{ }^{1} \mathrm{H}$ NMR $\left(\mathrm{C}_{6} \mathrm{D}_{6}\right)$ $\left.\delta_{\mathrm{H}} 3.06(3 \mathrm{H}, \mathrm{s}), 3.11(3 \mathrm{H}, \mathrm{s}), 3.17(1 \mathrm{H}, \mathrm{dd}, J) \quad 9.7,3.3 \mathrm{~Hz}\right), 3.22$ $(3 \mathrm{H}, \mathrm{s}), 3.29(3 \mathrm{H}, \mathrm{s}), 3.34(3 \mathrm{H}, \mathrm{s}), 3.44(3 \mathrm{H}, \mathrm{s}), 3.516(3 \mathrm{H}, \mathrm{s})$, $3.522(1 \mathrm{H}, \mathrm{dd}, J) \quad 10.1,6.1 \mathrm{~Hz}), 3.59(1 \mathrm{H}, \mathrm{dd}, J) \quad 10.6,1.6 \mathrm{~Hz})$, $3.68-3.73(3 \mathrm{H}, \mathrm{m}), 3.76(1 \mathrm{H}, \mathrm{dd}, J) \quad 9.7,8.9 \mathrm{~Hz}), 3.99(1 \mathrm{H}, \mathrm{dd}$, J) $10.1,8.9 \mathrm{~Hz}), 4.01(1 \mathrm{H}, \mathrm{m}), 4.10(1 \mathrm{H}, \mathrm{ddd}, J) \quad 10.2,5.3,1.2$ $\mathrm{Hz}), 4.60(1 \mathrm{H}, \mathrm{d}, J) 3.6 \mathrm{~Hz}), 4.74(1 \mathrm{H}, \mathrm{dd}, J) \quad 11.4,5.3 \mathrm{~Hz})$, $4.88(1 \mathrm{H}, \mathrm{dd}, J) \quad 11.8,1.6 \mathrm{~Hz}), 5.52(1 \mathrm{H}, \mathrm{d}, J) \quad 1.6 \mathrm{~Hz}), 6.78$ $(2 \mathrm{H}, \mathrm{m}), 7.30(2 \mathrm{H}, \mathrm{m}) ;{ }^{13} \mathrm{C} \mathrm{NMR}\left(100.6 \mathrm{MHz}, \mathrm{C}_{6} \mathrm{D}_{6}\right) \delta_{\mathrm{C}} 55.2\left(\mathrm{CH}_{3}\right)$, $57.1\left(\mathrm{CH}_{3}\right), 57.6\left(\mathrm{CH}_{3}\right), 58.5\left(\mathrm{CH}_{3}\right), 58.9\left(\mathrm{CH}_{3}\right), 60.3\left(\mathrm{CH}_{3}\right), 60.8$ $\left(\mathrm{CH}_{3}\right), 70.4(\mathrm{CH}), 72.7\left(\mathrm{CH}_{2}\right), 73.5(\mathrm{CH}), 77.0(\mathrm{CH}), 78.0\left(\mathrm{CH}_{2}\right)$, $78.1(\mathrm{CH}), 78.5(\mathrm{CH}), 81.7(\mathrm{CH}), 82.3(\mathrm{CH}), 83.2(\mathrm{CH}), 97.5(\mathrm{CH})$,

$100.7(\mathrm{CH}), 122.9(2 \times \mathrm{CH}), 129.5(2 \times \mathrm{C}), 133.5(2 \times \mathrm{CH})$, $163.2(2 \times \mathrm{C})$; MS (FAB) $\mathrm{m} / z$ (rel int) $608\left(\mathrm{M}^{+}+\mathrm{Na}, 17\right), 219$ (48), 187 (100); HRMS m/z calcd for $\mathrm{C}_{27} \mathrm{H}_{39} \mathrm{NNaO}_{13} 608.2319$, 
found 608.2330. Anal. Calcd for $\mathrm{C}_{27} \mathrm{H}_{39} \mathrm{NO}_{13}$ : C, 55.38; $\mathrm{H}, 6.71$; N, 2.39. Found: C, 55.45; H, 6.44; N, 2.10.

Reductive HAT of Methyl 2,3,4,6-Tetra- $O$-methyl-r-D-mannopyranosyl-(1f4)-2,3-di- $O$-methyl-6- $O$-phthalimido-r-D-glucopyranoside (18). Method A with $n$-Buz $\mathrm{SnH}$. A solution of phthalimide $18(100 \mathrm{mg}, 0.170 \mathrm{mmol})$ in dry benzene $(12 \mathrm{~mL})$ containing $n-\mathrm{Bu}_{3} \mathrm{SnH}(68 \mu \mathrm{L}, 0.255 \mathrm{mmol})$ and AIBN $(5.5 \mathrm{mg}$, $0.034 \mathrm{mmol}$ ) was heated at reflux temperature for $2.5 \mathrm{~h}$. After this time another portion of $n-\mathrm{Bu}_{3} \mathrm{SnH}(23 \mu \mathrm{L}, 0.086 \mathrm{mmol})$ and AIBN (5.5 $\mathrm{mg}, 0.034 \mathrm{mmol}$ ) were added and heating at reflux was continued for an additional $1 \mathrm{~h}$. After cooling to room temperature the reaction mixture was concentrated under reduced pressure. The residue was dissolved in $\mathrm{CH}_{3} \mathrm{CN}$, washed with $n$-hexane and the combined more polar extracts were concentrated under reduced pressure. Acetylation of the crude residue in dry pyridine $(3 \mathrm{~mL})$ containing $\mathrm{Ac}_{2} \mathrm{O}(1 \mathrm{~mL})$ at room temperature for $4 \mathrm{~h}$ gave after chromatotron chromatography (hexanes-EtOAc, 40:60) methyl 2,3,4,6-tetra- $O$-methyl-§-L-gulopyranosyl-(1f4)-6- $O$-acetyl-2,3-di$O$-methyl-R-D-glucopyranoside (21) (38 $\mathrm{mg}, 0.078 \mathrm{mmol}, 46 \%)$ and methyl 2,3,4,6-tetra- $O$-methyl-R-D-mannopyranosyl-(1f4)-6- $O$ acetyl-2,3-di- $O$-methyl-R-D-glucopyranoside (41S) $(14 \mathrm{mg}, 0.029$ mmol, 17\%) both as colorless oils. Compound 21: $[\mathrm{R}]_{\mathrm{D}}+89.6(c$, 0.24); IR $1739 \mathrm{~cm}^{-1}$; ${ }^{1} \mathrm{H}$ NMR $\left(\mathrm{C}_{6} \mathrm{D}_{6}\right) \delta_{\mathrm{H}} 1.75(3 \mathrm{H}, \mathrm{s}), 3.11(3 \mathrm{H}$, s), $3.136(3 \mathrm{H}, \mathrm{s}), 3.138(1 \mathrm{H}, \mathrm{dd}, J) \quad 8.9,3.6 \mathrm{~Hz}), 3.16(3 \mathrm{H}, \mathrm{s})$, $3.18(3 \mathrm{H}, \mathrm{s}), 3.28(1 \mathrm{H}, \mathrm{dd}, J)$ 3.6, $1.4 \mathrm{~Hz}), 3.29(3 \mathrm{H}, \mathrm{s}), 3.36$ $(3 \mathrm{H}, \mathrm{s}), 3.46(1 \mathrm{H}, \mathrm{dd}, J) \quad 8.1,3.1 \mathrm{~Hz}), 3.55(1 \mathrm{H}, \mathrm{dd}, J) \quad 9.2,5.9$ $\mathrm{Hz}), 3.67(1 \mathrm{H}, \mathrm{dd}, J) \quad 3.6,3.6 \mathrm{~Hz}), 3.68(1 \mathrm{H}, \mathrm{dd}, J) \quad 9.2,7.6$ $\mathrm{Hz}), 3.75$ (3H, s), 3.85-3.91 (2H, m), 4.00 (1H, ddd, $J$ ) 8.9, 6.7, $1.9 \mathrm{~Hz}), 4.14(1 \mathrm{H}, \mathrm{ddd}, J) \quad 7.6,6.2,1.4 \mathrm{~Hz}), 4.64(1 \mathrm{H}, \mathrm{dd}, J)$ 12.3, $6.7 \mathrm{~Hz}), 4.65(1 \mathrm{H}, \mathrm{d}, J) \quad 3.6 \mathrm{~Hz}), 4.83(1 \mathrm{H}, \mathrm{dd}, J) \quad 12.0$, $2.2 \mathrm{~Hz}), 5.43(1 \mathrm{H}, \mathrm{d}, J) \quad 8.1 \mathrm{~Hz}) ;{ }^{13} \mathrm{C} \mathrm{NMR}\left(\mathrm{C}_{6} \mathrm{D}_{6}\right) \delta_{\mathrm{C}} 20.6\left(\mathrm{CH}_{3}\right)$, $54.8\left(\mathrm{CH}_{3}\right), 58.1\left(\mathrm{CH}_{3}\right), 58.4\left(\mathrm{CH}_{3}\right), 58.8\left(\mathrm{CH}_{3}\right), 59.3\left(\mathrm{CH}_{3}\right), 59.5$ $\left(\mathrm{CH}_{3}\right), 61.1\left(\mathrm{CH}_{3}\right), 64.6\left(\mathrm{CH}_{2}\right), 68.9(\mathrm{CH}), 71.160\left(\mathrm{CH}_{2}\right), 71.9(\mathrm{CH})$, $76.3(\mathrm{CH}), 77.4(\mathrm{CH}), 77.574(\mathrm{CH}), 80.0(\mathrm{CH}), 82.5(\mathrm{CH}), 84.0$ $(\mathrm{CH}), 97.7(\mathrm{CH}), 101.6(\mathrm{CH}), 170.0(\mathrm{C}) ; \mathrm{MS} \mathrm{m} / \mathrm{z}$ (rel int) $450\left(\mathrm{M}^{+}\right.$

$\left.\mathrm{CH}_{3} \mathrm{OH},<1\right), 419$ (<1), 307 (48), 247 (100); HRMS m/z calcd for $\mathrm{C}_{20} \mathrm{H}_{34} \mathrm{O}_{11}$ 450.2101, found 450.2108. Anal. Calcd for $\mathrm{C}_{21} \mathrm{H}_{38} \mathrm{O}_{12}$ : C, 52.27; H, 7.94. Found: C, 52.28; H, 7.91.Compound 41S: $[R]_{\mathrm{D}}+62.3(c, 0.96)$; IR $1742 \mathrm{~cm}^{-1} ;{ }^{1} \mathrm{H}$ NMR $(400 \mathrm{MHz}$, $\left.\left.\mathrm{C}_{6} \mathrm{D}_{6}\right) \delta_{\mathrm{H}} 1.77(3 \mathrm{H}, \mathrm{s}), 3.06(1 \mathrm{H}, \mathrm{dd}, J) \quad 9.5,3.4 \mathrm{~Hz}\right), 3.08(3 \mathrm{H}$, s), $3.09(3 \mathrm{H}, \mathrm{s}), 3.28(3 \mathrm{H}, \mathrm{s}), 3.34(3 \mathrm{H}, \mathrm{s}), 3.36(3 \mathrm{H}, \mathrm{s}), 3.48(3 \mathrm{H}$, s), $3.51(3 \mathrm{H}, \mathrm{s}), 3.69-3.82(7 \mathrm{H}, \mathrm{m}), 3.89(1 \mathrm{H}, \mathrm{dd}, J) 9.8,9.8$ $\mathrm{Hz}), 4.01(1 \mathrm{H}, \mathrm{ddd}, J) \quad 9.8,5.0,1.9 \mathrm{~Hz}), 4.40(1 \mathrm{H}, \mathrm{dd}, J) \quad 12.2$, $4.8 \mathrm{~Hz}), 4.58(1 \mathrm{H}, \mathrm{d}, J) \quad 3.4 \mathrm{~Hz}), 4.67(1 \mathrm{H}, \mathrm{dd}, J) \quad 11.9,2.1 \mathrm{~Hz})$, $5.55(1 \mathrm{H}, \mathrm{d}, J) \quad 1.8 \mathrm{~Hz}) ;{ }^{13} \mathrm{C} \mathrm{NMR}\left(100.6 \mathrm{MHz}, \mathrm{C}_{6} \mathrm{D}_{6}\right) \delta_{\mathrm{C}} 20.5$ $\left(\mathrm{CH}_{3}\right), 54.8\left(\mathrm{CH}_{3}\right), 57.3\left(\mathrm{CH}_{3}\right), 57.6\left(\mathrm{CH}_{3}\right), 58.6\left(\mathrm{CH}_{3}\right), 59.1\left(\mathrm{CH}_{3}\right)$, $60.4\left(\mathrm{CH}_{3}\right), 60.8\left(\mathrm{CH}_{3}\right), 63.9\left(\mathrm{CH}_{2}\right), 68.8(\mathrm{CH}), 72.4\left(\mathrm{CH}_{2}\right), 73.8$ $(\mathrm{CH}), 76.9(\mathrm{CH}), 77.3(\mathrm{CH}), 78.2(\mathrm{CH}), 81.9(\mathrm{CH}), 82.6(\mathrm{CH})$, $83.8(\mathrm{CH}), 97.4(\mathrm{CH}), 100.2(\mathrm{CH}), 170.0(\mathrm{C}) ; \mathrm{MS} \mathrm{m} / \mathrm{z}$ (rel int) 419 $\left(\mathrm{M}^{+}-\mathrm{C}_{2} \mathrm{H}_{7} \mathrm{O}_{2}, 1\right), 405$ (1), 307 (100); HRMS $\mathrm{m} / \mathrm{z}$ calcd for $\mathrm{C}_{19} \mathrm{H}_{31} \mathrm{O}_{10} 419.1917$, found 419.1927. Anal. Calcd for $\mathrm{C}_{21} \mathrm{H}_{38} \mathrm{O}_{12}$ : C, 52.27; H, 7.94. Found: C, 52.25; H, 8.03.

Method B with $\boldsymbol{n}$-Bu $\mathbf{B n D}$. A solution of phthalimide $\mathbf{1 8}$ (82 $\mathrm{mg}, 0.140 \mathrm{mmol})$ in dry benzene $(10 \mathrm{~mL})$ containing $n-\mathrm{Bu}_{3} \mathrm{SnD}$ $(56 \mu \mathrm{L}, 0.210 \mathrm{mmol})$ and AIBN $(4.6 \mathrm{mg}, 0.028 \mathrm{mmol})$ was heated at reflux temperature for $2.5 \mathrm{~h}$. After this time another portion of $n$ - $\mathrm{Bu}_{3} \mathrm{SnD}(37 \mu \mathrm{L}, 0.138 \mathrm{mmol})$ and AIBN (4.6 mg, $\left.0.028 \mathrm{mmol}\right)$ were added and heating at reflux was continued for an additional $1 \mathrm{~h}$. After cooling to room temperature the reaction mixture was concentrated under reduced pressure. The residue was dissolved in $\mathrm{CH}_{3} \mathrm{CN}$, washed with $n$-hexane and the combined more polar extracts were concentrated under reduced pressure. Acetylation of the crude residue in dry pyridine $(3 \mathrm{~mL})$ containing $\mathrm{Ac}_{2} \mathrm{O}(1 \mathrm{~mL})$ at room temperature for $4 \mathrm{~h}$ after gave chromatotron chromatography (hexanes-EtOAc, 40:60) methyl 2,3,4,6-tetra- $O$-methyl-§L- $(5-2 \mathrm{H})$ gulopyranosyl-(1f4)-2,3-di- $O$-methyl-R-D-glucopyranoside [21-(D)] (35 mg, $0.072 \mathrm{mmol}, 52 \%$ ) and methyl 2,3,4,6-tetra$O$-methyl-R-D-[5-2 $\mathrm{H}]$ mannopyranosyl-(1f4)-2,3-di- $O$-methyl-R-Dglucopyranoside (41S-[D]) (14 mg, $0.029 \mathrm{mmol}, 21 \%,{ }^{1} \mathrm{H} /{ }^{2} \mathrm{H}$ ratio,
3:7) both as colorless oils. Compound 21-(D): ${ }^{1} \mathrm{H}$ NMR $\left(\mathrm{C}_{6} \mathrm{D}_{6}\right) \delta_{\mathrm{H}}$ $1.75(3 \mathrm{H}, \mathrm{s}), 3.11(3 \mathrm{H}, \mathrm{s}), 3.136(3 \mathrm{H}, \mathrm{s}), 3.137(1 \mathrm{H}, \mathrm{dd}, J)$ 9.2, $3.6 \mathrm{~Hz}), 3.16(3 \mathrm{H}, \mathrm{s}), 3.18(3 \mathrm{H}, \mathrm{s}), 3.28(1 \mathrm{H}, \mathrm{d}, J) \quad 3.6 \mathrm{~Hz}), 3.29$ $(3 \mathrm{H}, \mathrm{s}), 3.36(3 \mathrm{H}, \mathrm{s}), 3.45(1 \mathrm{H}, \mathrm{dd}, J) \quad 8.1,2.8 \mathrm{~Hz}), 3.55(1 \mathrm{H}, \mathrm{d}$, $J) \quad 9.2 \mathrm{~Hz}), 3.67(1 \mathrm{H}, \mathrm{dd}, J) \quad 3.6,3.4 \mathrm{~Hz}), 3.68(1 \mathrm{H}, \mathrm{d}, J) 9.5$ $\mathrm{Hz}), 3.75$ (3H, s), 3.85-3.91 (2H, m), 4.00 (1H, ddd, $J) \quad 8.9,6.7$, $2.2 \mathrm{~Hz}), 4.64(1 \mathrm{H}, \mathrm{dd}, J) \quad 12.3,6.7 \mathrm{~Hz}), 4.65(1 \mathrm{H}, \mathrm{d}, J) \quad 3.6 \mathrm{~Hz})$, $4.83(1 \mathrm{H}, \mathrm{dd}, J) \quad 11.8,2.2 \mathrm{~Hz}), 5.43(1 \mathrm{H}, \mathrm{d}, J) 8.1 \mathrm{~Hz}) ;{ }^{13} \mathrm{C}$ NMR $\left(\mathrm{C}_{6} \mathrm{D}_{6}\right) \delta_{\mathrm{C}} 20.6\left(\mathrm{CH}_{3}\right), 54.8\left(\mathrm{CH}_{3}\right), 58.1\left(\mathrm{CH}_{3}\right), 58.4\left(\mathrm{CH}_{3}\right)$, $58.8\left(\mathrm{CH}_{3}\right), 59.3\left(\mathrm{CH}_{3}\right), 59.5\left(\mathrm{CH}_{3}\right), 61.1\left(\mathrm{CH}_{3}\right), 64.6\left(\mathrm{CH}_{2}\right), 68.9$ $(\mathrm{CH}), 71.091\left(\mathrm{CH}_{2}\right), 76.3(\mathrm{CH}), 77.47(\mathrm{CH}), 77.522(\mathrm{CH}), 80.0$ $(\mathrm{CH}), 82.5(\mathrm{CH}), 84.0(\mathrm{CH}), 97.7(\mathrm{CH}), 101.6(\mathrm{CH}), 170.0(\mathrm{C})$; MS m/z (rel int) $452\left(\mathrm{M}^{+}-\mathrm{CH}_{3} \mathrm{O},<1\right), 307$ (43), 247 (70), 88 (100); HRMS $\mathrm{m} / \mathrm{z}$ calcd for $\mathrm{C}_{2} \mathrm{H} 2 \mathrm{H} / \mathrm{O}$ 452.2242, found 452.2242. Compund 41S-[D]: ${ }^{1} \mathrm{H}$ NMR $\left(400 \mathrm{MHz}, \mathrm{C}_{6} \mathrm{D}_{6}\right) \delta_{\mathrm{H}} 1.77(3 \mathrm{H}, \mathrm{s})$, $3.06(1 \mathrm{H}, \mathrm{dd}, J)$ 9.5, $3.4 \mathrm{~Hz}), 3.08(3 \mathrm{H}, \mathrm{s}), 3.09(3 \mathrm{H}, \mathrm{s}), 3.28$ $(3 \mathrm{H}, \mathrm{s}), 3.34(3 \mathrm{H}, \mathrm{s}), 3.36(3 \mathrm{H}, \mathrm{s}), 3.48(3 \mathrm{H}, \mathrm{s}), 3.51(3 \mathrm{H}, \mathrm{s})$, 3.69-3.82 (7H, m), $3.89(1 \mathrm{H}, \mathrm{d}, J) 9.0 \mathrm{~Hz}), 3.90(1 \mathrm{H}, \mathrm{dd}, J)$ $9.8,9.8 \mathrm{~Hz}), 4.01(1 \mathrm{H}, \mathrm{ddd}, J) \quad 9.8,5.0,1.9 \mathrm{~Hz}), 4.40(1 \mathrm{H}, \mathrm{dd}, J$ ) $12.2,4.8 \mathrm{~Hz}), 4.58(1 \mathrm{H}, \mathrm{d}, J) \quad 3.4 \mathrm{~Hz}), 4.67(1 \mathrm{H}, \mathrm{dd}, J) \quad 11.9$, $2.1 \mathrm{~Hz}), 5.55(1 \mathrm{H}, \mathrm{d}, J) \quad 1.8 \mathrm{~Hz}) ;{ }^{13} \mathrm{C} \mathrm{NMR}\left(125.7 \mathrm{MHz}, \mathrm{C}_{6} \mathrm{D}_{6}\right)$ $\delta_{\mathrm{C}} 20.5\left(\mathrm{CH}_{3}\right), 54.8\left(\mathrm{CH}_{3}\right), 57.3\left(\mathrm{CH}_{3}\right), 57.6\left(\mathrm{CH}_{3}\right), 58.6\left(\mathrm{CH}_{3}\right)$, $59.1\left(\mathrm{CH}_{3}\right), 60.4\left(\mathrm{CH}_{3}\right), 60.8\left(\mathrm{CH}_{3}\right), 63.9\left(\mathrm{CH}_{2}\right), 68.8(\mathrm{CH}), 72.312$ $\left(\mathrm{CH}_{2}\right), 72.376\left(\mathrm{CH}_{2}\right), 73.8(\mathrm{CH}), 76.894(\mathrm{CH}), 76.947(\mathrm{CH}), 77.3$ $(\mathrm{CH}), 78.3(\mathrm{CH}), 81.9(\mathrm{CH}), 82.6(\mathrm{CH}), 83.8(\mathrm{CH}), 97.4(\mathrm{CH})$, $100.2(\mathrm{CH}), 170.0(\mathrm{C}) ; \mathrm{MS} \mathrm{m} / \mathrm{z}$ (rel int) $451\left(\mathrm{M}^{+}-\mathrm{CH}_{3} \mathrm{OH},<1\right)$, 450 (<1), 307 (19), 247 (26), 88 (100); HRMS m/z calcd for $\mathrm{C}_{20} \mathrm{H}_{34}{ }^{2} \mathrm{HO}_{11} 451.2164$, found 451.2179 .

Methyl 2,3,4,6-Tetra- $O$-acetyl-r-D-mannopyranosyl-(1f4)-2,3di- $\boldsymbol{O}$-methyl-6- $\boldsymbol{O}$-phthalimido-r-D-glucopyranoside (20). DEAD (192 $\mu \mathrm{L}, 1.22 \mathrm{mmol}$ ) was added dropwise to a stirred solution of the alcohol 19 (270 mg, $0.489 \mathrm{mmol}), N$-hydroxyphthalimide (199 $\mathrm{mg}, 1.22 \mathrm{mmol})$ and $\mathrm{PPh}_{3}(320 \mathrm{mg}, 1.22 \mathrm{mmol})$ in dry THF (5.3 $\mathrm{mL}$ ) and the resulting solution was stirred at $0{ }^{\circ} \mathrm{C}$ for $2 \mathrm{~h}$. Then the solvent was removed and the crude was quenched with water and extracted with $\mathrm{CHCl}_{3}$. The combined extracts were dried over $\mathrm{Na}_{2} \mathrm{SO}_{4}$ and concentrated under reduced pressure. The residue obtained was purified by column chromatography (hexanes-EtOAc, 70:30 f 30:70) to give compound 20 (284 mg, $0.407 \mathrm{mmol}, 83 \%)$ as a white foam: $[R]_{\mathrm{D}}+72.9(c, 0.122)$; IR 1791, $1734 \mathrm{~cm}^{-1} ;{ }^{1} \mathrm{H}$ NMR $\delta_{\mathrm{H}} 1.98(3 \mathrm{H}, \mathrm{s}), 2.04(3 \mathrm{H}, \mathrm{s}), 2.06(3 \mathrm{H}, \mathrm{s}), 2.13(3 \mathrm{H}, \mathrm{s}), 3.25$ $(1 \mathrm{H}, \mathrm{dd}, J) \quad 9.8,3.5 \mathrm{~Hz}), 3.41(3 \mathrm{H}, \mathrm{s}), 3.48(3 \mathrm{H}, \mathrm{s}), 3.59(1 \mathrm{H}, \mathrm{dd}$, $J) \quad 8.6,8.6 \mathrm{~Hz}), 3.61(3 \mathrm{H}, \mathrm{s}), 3.88(1 \mathrm{H}, \mathrm{ddd}, J) \quad 10.0,2.6,2.6$ $\mathrm{Hz}), 3.95(1 \mathrm{H}, \mathrm{dd}, J) \quad 10.0,8.6 \mathrm{~Hz}), 4.07(1 \mathrm{H}, \mathrm{dd}, J) \quad 11.8,2.3$ $\mathrm{Hz}), 4.11(1 \mathrm{H}, \mathrm{ddd}, J) \quad 9.2,5.5,2.0 \mathrm{~Hz}), 4.23(1 \mathrm{H}, \mathrm{dd}, J) \quad 12.1$, $5.5 \mathrm{~Hz}), 4.43-4.48(2 \mathrm{H}, \mathrm{m}), 4.71(1 \mathrm{H}, \mathrm{d}, J) \quad 3.4 \mathrm{~Hz}), 5.260(1 \mathrm{H}$ d, $J) \quad 2.0 \mathrm{~Hz}), 5.262(1 \mathrm{H}, \mathrm{dd}, J) 9.8,9.8 \mathrm{~Hz}), 5.31(1 \mathrm{H}, \mathrm{dd}, J)$ 9.8, 3.2 Hz), $5.36(1 \mathrm{H}, \mathrm{dd}, J) \quad 3.2,2.0 \mathrm{~Hz}), 7.73(2 \mathrm{H}, \mathrm{m}), 7.81$ $(2 \mathrm{H}, \mathrm{m}) ;{ }^{13} \mathrm{C} \mathrm{NMR}(100.6 \mathrm{MHz}) \delta_{\mathrm{C}} 20.58\left(\mathrm{CH}_{3}\right), 20.63\left(\mathrm{CH}_{3}\right)$, $20.7\left(\mathrm{CH}_{3}\right), 20.8\left(\mathrm{CH}_{3}\right), 55.6\left(\mathrm{CH}_{3}\right), 58.8\left(\mathrm{CH}_{3}\right), 61.1\left(\mathrm{CH}_{3}\right), 62.8$ $\left(\mathrm{CH}_{2}\right), 66.1(\mathrm{CH}), 69.0(\mathrm{CH}), 69.36(\mathrm{CH}), 69.43(\mathrm{CH}), 69.6(\mathrm{CH})$, $76.4\left(\mathrm{CH}_{2}\right), 77.7(\mathrm{CH}), 81.8(\mathrm{CH}), 82.6(\mathrm{CH}), 97.5(\mathrm{CH}), 99.9(\mathrm{CH})$, $123.4(2 \times \mathrm{CH}), 128.9(2 \times \mathrm{C}), 134.4(2 \times \mathrm{CH}), 163.1(2 \times \mathrm{C})$, 169.7 (C), 169.8 (C), 169.9 (C), 170.7 (C); MS m/z (rel int) 577 $\left(\mathrm{M}^{+}\right.$- 2AcOH, <1), $492(<1), 417$ (1), 331 (70), 88 (100); HRMS $\mathrm{m} / \mathrm{z}$ calcd for $\mathrm{C}_{27} \mathrm{H}_{31} \mathrm{NO}_{13} 577.1795$, found 577.1814. Anal. Calcd for $\mathrm{C}_{31} \mathrm{H}_{39} \mathrm{NO}_{17}$ : C, 53.37; H, 5.63; N, 2.01. Found: C, 53.61; H, $5.62 ; \mathrm{N}, 1.80$.

Reductive HAT of Methyl 2,3,4,6-Tetra- $O$-acetyl-r-D-mannopyranosyl-(1f4)-2,3-di- $O$-methyl-6- $O$-phthalimido-r-D-glucopyranoside (20). Method A with $\boldsymbol{n}-\mathrm{Bu}_{3} \mathrm{SnH}$. A solution of phthalimide $20(80 \mathrm{mg}, 0.114 \mathrm{mmol})$ in dry benzene $(8 \mathrm{~mL})$ containing $n-\mathrm{Bu}_{3} \mathrm{SnH}(37 \mu \mathrm{L}, 0.136 \mathrm{mmol})$ and AIBN (2 mg, 0.011 $\mathrm{mmol}$ ) was heated at reflux temperature for $2 \mathrm{~h}$. After this time another portion of $n-\mathrm{Bu}_{3} \mathrm{SnH}(15 \mu \mathrm{L}, 0.057 \mathrm{mmol})$ and AIBN (2 $\mathrm{mg}, 0.011 \mathrm{mmol}$ ) were added and heating at reflux was continued for an additional $1 \mathrm{~h}$. After cooling to room temperature the reaction mixture was concentrated under reduced pressure. The residue was dissolved in $\mathrm{CH}_{3} \mathrm{CN}$, washed with $n$-hexane and the combined more 
polar extracts were concentrated under reduced pressure. Column chromatography of the crude residue (hexanes-EtOAc, 80:20 f 20:80) afforded methyl 2,3,4,6-tetra- $O$-acetyl-R-D-mannopyranosyl(1f4)-2,3-di-O-methyl-R-D-glucopyranoside (19) $(26.7 \mathrm{mg}, 0.048$ mmol, 42\%) and methyl 2,3,4,6-tetra- $O$-acetyl- $\$$-L-gulopyranosyl(1f4)-2,3-di- $O$-methyl-R-D-glucopyranoside (22) (27 mg, 0.049 $\mathrm{mmol}, 43 \%)$, as colorless oils. Compound 22: $[\mathrm{R}]_{\mathrm{D}}+66.2(c, 0.12)$; IR 3544, $1750 \mathrm{~cm}^{-1} ; \quad{ }^{1} \mathrm{H}$ NMR $\delta_{\mathrm{H}} 2.01(3 \mathrm{H}, \mathrm{s}), 2.10(3 \mathrm{H}, \mathrm{s}), 2.13$ $(3 \mathrm{H}, \mathrm{s}), 2.17(3 \mathrm{H}, \mathrm{s}), 2.70(1 \mathrm{H}, \mathrm{dd}, J) \quad 9.0,5.5 \mathrm{~Hz}), 3.24(1 \mathrm{H}, \mathrm{dd}$, $J$ ) $9.5,3.5 \mathrm{~Hz}), 3.41(3 \mathrm{H}, \mathrm{s}), 3.52(3 \mathrm{H}, \mathrm{s}), 3.54(1 \mathrm{H}, \mathrm{m}), 3.55$ $(1 \mathrm{H}, \mathrm{dd}, J) \quad 9.3,9.3 \mathrm{~Hz}), 3.62(3 \mathrm{H}, \mathrm{s}), 3.67(1 \mathrm{H}, \mathrm{ddd}, J) \quad 12.5$, 9.1, $2.0 \mathrm{~Hz}), 3.74(1 \mathrm{H}, \mathrm{dd}, J) \quad 9.5,9.5 \mathrm{~Hz}), 3.97(1 \mathrm{H}, \mathrm{dd}, J)$ 11.0, $8.0 \mathrm{~Hz}), 4.03(1 \mathrm{H}$, ddd, $J) \quad 12.5,6.0,2.5 \mathrm{~Hz}), 4.23-4.29$ $(2 \mathrm{H}, \mathrm{m}), 4.86(1 \mathrm{H}, \mathrm{d}, J) \quad 3.5 \mathrm{~Hz}), 4.92(1 \mathrm{H}, \mathrm{d}, J) \quad 3.5 \mathrm{~Hz}), 4.95$ $(1 \mathrm{H}, \mathrm{dd}, J) \quad 8.5,3.5 \mathrm{~Hz}), 5.21(1 \mathrm{H}, \mathrm{d}, J) \quad 8.5 \mathrm{~Hz}), 5.38(1 \mathrm{H}, \mathrm{dd}$, J) $3.5,3.5 \mathrm{~Hz}) ;{ }^{13} \mathrm{C} \mathrm{NMR} \delta_{\mathrm{C}} 20.6\left(\mathrm{CH}_{3}\right), 20.7\left(3 \times \mathrm{CH}_{3}\right), 55.2$ $\left(\mathrm{CH}_{3}\right), 58.9\left(\mathrm{CH}_{3}\right), 60.8\left(\mathrm{CH}_{2}\right), 61.1\left(\mathrm{CH}_{3}\right), 62.560\left(\mathrm{CH}_{2}\right), 67.8$ $(\mathrm{CH}), 68.122(\mathrm{CH}), 68.4(\mathrm{CH}), 70.0(\mathrm{CH}), 70.9(\mathrm{CH}), 74.9(\mathrm{CH})$, $82.3(\mathrm{CH}), 83.1(\mathrm{CH}), 97.5(\mathrm{CH}), 99.1(\mathrm{CH}), 168.8(\mathrm{C}), 169.36$ (C), $169.44(\mathrm{C}), 170.6(\mathrm{C})$; MS m/z (rel int) $492\left(\mathrm{M}^{+}-\mathrm{AcOH}\right.$, $<1$ ), 331 (35), 169 (26), 88 (100); HRMS m/z calcd for $\mathrm{C}_{21} \mathrm{H}_{32} \mathrm{O}_{13}$ 492.1843, found 492.1826. Anal. Calcd for $\mathrm{C}_{23} \mathrm{H}_{36} \mathrm{O}_{15}: \mathrm{C}, 50.00$; H, 6.57. Found: C, 50.06; H, 6.72.

Method B with $\boldsymbol{n}$-Bus $\mathbf{S n D}$. A solution of phthalimide 20 (129 $\mathrm{mg}, 0.185 \mathrm{mmol})$ in dry benzene $(13 \mathrm{~mL})$ containing $n-\mathrm{Bu}_{3} \mathrm{SnH}$ (55 $\mu \mathrm{L}, 0.277 \mathrm{mmol}$ ) and AIBN (3 mg, $0.018 \mathrm{mmol}$ ) was heated at reflux temperature for $2 \mathrm{~h}$. After this time another portion of $n$ $\mathrm{Bu}_{3} \mathrm{SnD}(55 \mu \mathrm{L}, 0.277 \mathrm{mmol})$ and AIBN (3 mg, $\left.0.018 \mathrm{mmol}\right)$ was added and heating at reflux continued for an additional $1 \mathrm{~h}$. After cooling to room temperature the reaction mixture was concentrated under reduced pressure. The residue was dissolved in $\mathrm{CH}_{3} \mathrm{CN}$, washed with $n$-hexane and the combined more polar extracts were concentrated under reduced pressure. Column chromatography of the crude residue (hexanes-EtOAc, 80:20 f 20: 80) afforded methyl 2,3,4,6-tetra- $O$-acetyl-R-D-[5-2 $\mathrm{H}]$ mannopyranosyl(1f4)-2,3-di- $O$-methyl-R-D-glucopyranoside (19-[D]) (30 mg, 0.054 mmol, $29 \%,{ }^{1} \mathrm{H} / 2 \mathrm{H}$ ratio, 3:7) and methyl 2,3,4,6-tetra- $O$-acetyl$\S$-L-(5-2H)gulopyranosyl-(1 f4)-2,3-di- $O$-methyl-R-D-glucopyranoside [22-(D)] (40.2 mg, $0.072 \mathrm{mmol}, 39 \%$ ), as colorless oils. Compound 19-[D]: ${ }^{1} \mathrm{H}$ NMR $\delta_{\mathrm{H}} 1.99(3 \mathrm{H}, \mathrm{s}), 2.04(3 \mathrm{H}, \mathrm{s}), 2.11$ $(3 \mathrm{H}, \mathrm{s}), 2.14(3 \mathrm{H}, \mathrm{s}), 3.19(1 \mathrm{H}, \mathrm{dd}, J) \quad 9.3,3.7 \mathrm{~Hz}), 3.42(3 \mathrm{H}, \mathrm{s})$, $3.49(3 \mathrm{H}, \mathrm{s}), 3.58(1 \mathrm{H}, \mathrm{dd}, J) 8.9,8.9 \mathrm{~Hz}), 3.59(3 \mathrm{H}, \mathrm{s}), 3.62-3.65$ $(2 \mathrm{H}, \mathrm{m}), 3.81(2 \mathrm{H}, \mathrm{m}), 4.05(1 \mathrm{H}, \mathrm{ddd}, J) \quad 8.9,6.1,2.0 \mathrm{~Hz}), 4.134$ $(1 \mathrm{H}, \mathrm{d}, J) 12.1 \mathrm{~Hz}), 4.137(1 \mathrm{H}, \mathrm{dd}, J) 12.1,2.8 \mathrm{~Hz}), 4.210(1 \mathrm{H}$, d, $J) \quad 12.2 \mathrm{~Hz}), 4.215(1 \mathrm{H}, \mathrm{d}, J) \quad 12.2,4.5 \mathrm{~Hz}), 4.81(1 \mathrm{H}, \mathrm{dd}, J$ ) $3.6 \mathrm{~Hz}), 5.236(1 \mathrm{H}, \mathrm{d}, J) \quad 9.7 \mathrm{~Hz}), 5.238(1 \mathrm{H}, \mathrm{dd}, J) \quad 9.7,9.7$ $\mathrm{Hz}), 5.25(1 \mathrm{H}, \mathrm{d}, J) \quad 1.6 \mathrm{~Hz}), 5.30(1 \mathrm{H}, \mathrm{dd}, J) \quad 9.8,3.2 \mathrm{~Hz}), 5.31$ $(1 \mathrm{H}, \mathrm{dd}, J) 3.2,1.6 \mathrm{~Hz}) .{ }^{13} \mathrm{C} \mathrm{NMR} \delta_{\mathrm{C}} 20.6\left(3 \times \mathrm{CH}_{3}\right), 20.8\left(\mathrm{CH}_{3}\right)$, $55.3\left(\mathrm{CH}_{3}\right), 58.9\left(\mathrm{CH}_{3}\right), 61.3\left(\mathrm{CH}_{3}\right), 61.5\left(\mathrm{CH}_{2}\right), 62.942\left(\mathrm{CH}_{2}\right)$, $63.011\left(\mathrm{CH}_{2}\right), 62.235(\mathrm{CH}), 66.287(\mathrm{CH}), 68.8(\mathrm{CH}), 69.5(\mathrm{CH})$, $69.6(\mathrm{CH}), 70.0(\mathrm{CH}), 76.6(\mathrm{CH}), 82.4(\mathrm{CH}), 83.0(\mathrm{CH}), 97.5(\mathrm{CH})$, $99.5(\mathrm{CH}), 169.7(\mathrm{C}), 169.8(\mathrm{C}), 169.9(\mathrm{C}), 170.7(\mathrm{C}) ; \mathrm{MS} \mathrm{m} / z$ (rel int) $493\left(\mathrm{M}^{+}\right.$- $\left.\mathrm{AcOH}, 2\right), 492(<1), 332$ (44), 331 (15), 88 (100); HRMS $m / z$ calcd for $\mathrm{C}_{21} \mathrm{H}_{31}{ }^{2} \mathrm{HO}_{13} 493.1906$, found 493.1914 . Compound 22-(D): ${ }^{1} \mathrm{H}$ NMR $\delta_{\mathrm{H}} 2.00(3 \mathrm{H}, \mathrm{s}), 2.08(3 \mathrm{H}, \mathrm{s}), 2.12$ $(3 \mathrm{H}, \mathrm{s}), 2.16(3 \mathrm{H}, \mathrm{s}), 2.69(1 \mathrm{H}, \mathrm{dd}, J) \quad 8.9,6.1 \mathrm{~Hz}, \mathrm{OH}), 3.22$ $(1 \mathrm{H}, \mathrm{dd}, J) \quad 9.3,3.6 \mathrm{~Hz}), 3.40(3 \mathrm{H}, \mathrm{s}), 3.506(3 \mathrm{H}, \mathrm{s}), 3.510(1 \mathrm{H}$, m), $3.55(1 \mathrm{H}, \mathrm{dd}, J) \quad 9.4,9.4 \mathrm{~Hz}), 3.61(3 \mathrm{H}, \mathrm{s}), 3.66(1 \mathrm{H}, \mathrm{ddd}, J$ ) $12,6,8.9,2.0 \mathrm{~Hz}), 3.73(1 \mathrm{H}, \mathrm{dd}, J) \quad 9.4,9.4 \mathrm{~Hz}), 3.96(1 \mathrm{H}, \mathrm{d}$, $J) \quad 11.8 \mathrm{~Hz}), 4.01(1 \mathrm{H}, \mathrm{ddd}, J) \quad 13.0,6.1,2.4 \mathrm{~Hz}), 4.26(1 \mathrm{H}, \mathrm{d}$, J) $11.8 \mathrm{~Hz}), 4.85(1 \mathrm{H}, \mathrm{d}, J) 3.6 \mathrm{~Hz}), 4.90(1 \mathrm{H}, \mathrm{d}, J) 3.7 \mathrm{~Hz})$, $4.94(1 \mathrm{H}, \mathrm{dd}, J)$ 8.5, 3.2 Hz), $5.20(1 \mathrm{H}, \mathrm{d}, J) \quad 8.5 \mathrm{~Hz}), 5.37(1 \mathrm{H}$, $\mathrm{dd}, J) \quad 3.2,3.2 \mathrm{~Hz}) ;{ }^{13} \mathrm{C} \mathrm{NMR} \delta_{\mathrm{C}} 20.5\left(\mathrm{CH}_{3}\right), 20.6\left(3 \times \mathrm{CH}_{3}\right)$, $55.2\left(\mathrm{CH}_{3}\right), 58.9\left(\mathrm{CH}_{3}\right), 60.7\left(\mathrm{CH}_{2}\right), 61.0\left(\mathrm{CH}_{3}\right), 62.439\left(\mathrm{CH}_{2}\right)$, $67.8(\mathrm{CH}), 68.021(\mathrm{CH}), 68.4(\mathrm{CH}), 69.9(\mathrm{CH}), 74.9(\mathrm{CH}), 82.2$ $(\mathrm{CH}), 83.1(\mathrm{CH}), 97.5(\mathrm{CH}), 99.0(\mathrm{CH}), 168.8(\mathrm{C}), 169.3(\mathrm{C}), 169.4$ (C), 170.5 (C); MS m/z (rel int) $493\left(\mathrm{M}^{+}\right.$- $\left.\mathrm{AcOH}, 2\right), 332$ (59), 170 (36), 88 (100); HRMS m/z calcd for $\mathrm{C}_{21} \mathrm{H}_{31}{ }^{2} \mathrm{HO}_{13} 493.1906$, found 493.1894 .
Methyl 2,3,4-Tri- $O$-methyl-r-L-rhamnopyranosyl-(1f4)-2,3-di$\boldsymbol{O}$-methyl-6- $\boldsymbol{O}$-phthalimido-r-D-galactopyranoside (24). DEAD (234 $\mu \mathrm{L}, 1.462 \mathrm{mmol}$ ) was added dropwise to a stirred solution of the alcohol 23 (300 mg, $0.732 \mathrm{mmol}$ ), $N$-hydroxyphthalimide (239 mg, $1.464 \mathrm{mmol})$ and $\mathrm{PPh}_{3}(383 \mathrm{mg}, 1.464 \mathrm{mmol})$ in dry THF $(8 \mathrm{~mL})$ and the resulting solution was stirred at $0{ }^{\circ} \mathrm{C}$ for $3 \mathrm{~h}$. Then the solvent was removed and the crude was quenched with water and extracted with $\mathrm{Et}_{2} \mathrm{O}$. The combined extracts were dried over $\mathrm{Na}_{2} \mathrm{SO}_{4}$ and concentrated under reduced pressure. The residue obtained was purified by column chromatography (hexanes-AcOEt, 50:50 f 30:70) to give compound 24 (331 $\mathrm{mg}, 0.596 \mathrm{mmol}, 82 \%)$ as a foam: $[\mathrm{R}]_{\mathrm{D}}+17.5(c, 0.12)$; IR 1793, $1739 \mathrm{~cm}^{-1} ;{ }^{1} \mathrm{H}$ NMR $(400 \mathrm{MHz})$ $\begin{array}{lll}\delta_{\mathrm{H}} 1.21(3 \mathrm{H}, \mathrm{d}, J) & 6.1 \mathrm{~Hz}), 3.08(1 \mathrm{H}, \mathrm{dd}, J) \quad 9.4,9.4 \mathrm{~Hz}), 3.42\end{array}$ $(1 \mathrm{H}, \mathrm{dd}, J) \quad 9.3,3.2 \mathrm{~Hz}), 3.45(3 \mathrm{H}, \mathrm{s}), 3.47(3 \mathrm{H}, \mathrm{s}), 3.48(3 \mathrm{H}, \mathrm{s})$, $3.496(3 \mathrm{H}, \mathrm{s}), 3.501(6 \mathrm{H}, \mathrm{s}), 3.54(1 \mathrm{H}, \mathrm{m}), 3.56(1 \mathrm{H}, \mathrm{dd}, J) \quad 9.3$, $2.9 \mathrm{~Hz}), 3.61(1 \mathrm{H}, \mathrm{dd}, J) \quad 10.1,2.6 \mathrm{~Hz}), 3.71(1 \mathrm{H}, \mathrm{dd}, J) \quad 3.4$, $2.1 \mathrm{~Hz}), 4.15(1 \mathrm{H}$, ddd, $J) \quad 5.6,5.6,0 \mathrm{~Hz}), 4.31(1 \mathrm{H}, \mathrm{m}), 4.33$ $(2 \mathrm{H}, \mathrm{d}, J) 5.6 \mathrm{~Hz}), 4.90(1 \mathrm{H}, \mathrm{d}, J) \quad 3.2 \mathrm{~Hz}), 5.15(1 \mathrm{H}, \mathrm{d}, J) 1.9$ $\mathrm{Hz}), 7.73-7.76(2 \mathrm{H}, \mathrm{m}), 7.82-7.84(2 \mathrm{H}, \mathrm{m}) ;{ }^{13} \mathrm{C}$ NMR $(100.6$ $\mathrm{MHz}) \delta_{\mathrm{C}} 17.6\left(\mathrm{CH}_{3}\right), 55.7\left(\mathrm{CH}_{3}\right), 57.6\left(\mathrm{CH}_{3}\right), 58.48\left(\mathrm{CH}_{3}\right), 58.53$ $\left(\mathrm{CH}_{3}\right), 58.67\left(\mathrm{CH}_{3}\right), 60.7\left(\mathrm{CH}_{3}\right), 68.2(\mathrm{CH}), 68.8(\mathrm{CH}), 74.0(\mathrm{CH})$, $77.4(\mathrm{CH}), 77.7(\mathrm{CH}), 78.3\left(\mathrm{CH}_{2}\right), 80.0(\mathrm{CH}), 80.6(\mathrm{CH}), 81.8(\mathrm{CH})$, $98.0(\mathrm{CH}), 98.5(\mathrm{CH}), 123.6(2 \times \mathrm{CH}), 128.9(2 \times \mathrm{C}), 134.5(2 \times$ $\mathrm{CH}), 163.4(2 \times \mathrm{C}) ; \mathrm{MS} \mathrm{m} / z(\%) 554\left(\mathrm{M}^{+}-\quad \mathrm{H}, 4\right), 524$ (6), 492 (100); HRMS $m / z$ calcd for $\mathrm{C}_{26} \mathrm{H}_{36} \mathrm{NO}_{12} 554.2238$, found 554.2216. Anal. Calcd for $\mathrm{C}_{26} \mathrm{H}_{37} \mathrm{NO}_{12}$ : C, 56.21; H, 6.71; N, 2.52. Found: C, 56.40; H, 6.98; N, 2.40.

Reductive HAT of Methyl 2,3,4-Tri- $O$-methyl-r-L-rhamnopyranosyl-(1f4)-2,3-di- $O$-methyl-6- $O$-phthalimido-r-D-glucopyranoside (24). Method A with $\boldsymbol{n}$-Bu $\mathbf{B} \mathrm{SnH}$. A solution of phthalimide 24 (40 $\mathrm{mg}, 0.072 \mathrm{mmol})$ in dry benzene $(5.5 \mathrm{~mL})$ containing $n$-Bu 3 SnH (19 $\mu \mathrm{L}, 0.072 \mathrm{mmol})$ and AIBN (1.2 mg, $0.007 \mathrm{mmol})$ was heated at reflux temperature for $1 \mathrm{~h}$. After cooling to room temperature the reaction mixture was concentrated under reduced pressure. The residue was dissolved in $\mathrm{CH}_{3} \mathrm{CN}$, washed with $n$ hexane and the combined more polar extracts were concentrated under reduced pressure. Column chromatography (hexanes-EtOAc, $50: 50 \mathrm{f} \quad 30: 70)$ gave methyl 6-deoxy-2,3,4-tri- $O$-methyl- $\$$-Dgulopyranosyl-(1f4)-2,3-di- $O$-methyl-R-D-galactopyranoside (27) (18 mg, $0.044 \mathrm{mmol}, 61 \%)$ and the alcohol 23 (4.5 mg, $0.011 \mathrm{mmol}$, $15 \%)$, both as colorless oils. Compound 27: $[\mathrm{R}]_{\mathrm{D}}+20.0(c, 0.18)$; IR $3482 \mathrm{~cm}^{-1}$; ${ }^{1} \mathrm{H}$ NMR (400 MHz) $\left.\delta_{\mathrm{H}} 1.25(3 \mathrm{H}, \mathrm{d}, J) \quad 6.6 \mathrm{~Hz}\right)$, $3.08(1 \mathrm{H}, \mathrm{dd}, J) \quad 3.4,1.6 \mathrm{~Hz}), 3.27(1 \mathrm{H}, \mathrm{dd}, J) \quad 8.0,3.2 \mathrm{~Hz})$, $3.39(3 \mathrm{H}, \mathrm{s}), 3.45(3 \mathrm{H}, \mathrm{s}), 3.47(3 \mathrm{H}, \mathrm{s}), 3.49(3 \mathrm{H}, \mathrm{s}), 3.50(3 \mathrm{H}, \mathrm{s})$, $3.55(1 \mathrm{H}, \mathrm{m}), 3.57(1 \mathrm{H}, \mathrm{dd}, J) \quad 10.1,2.9 \mathrm{~Hz}), 3.60(3 \mathrm{H}, \mathrm{s}), 3.62$ $(1 \mathrm{H}, \mathrm{dd}, J) \quad 10.1,3.2 \mathrm{~Hz}), 3.73(1 \mathrm{H}, \mathrm{dd}, J) 3.2,3.2 \mathrm{~Hz})$, 3.75-3.82 (2H, m), $3.96(1 \mathrm{H}$, dddd, $J)$ 6.4, 6.4, 6.4, $1.6 \mathrm{~Hz})$, $4.26(1 \mathrm{H}, \mathrm{dd}, J) \quad 2.7,0 \mathrm{~Hz}), 4.81(1 \mathrm{H}, \mathrm{d}, J) \quad 3.2 \mathrm{~Hz}), 4.90(1 \mathrm{H}$, $\mathrm{d}, J) \quad 8.0 \mathrm{~Hz}) ;{ }^{13} \mathrm{C} \mathrm{NMR}(100.6 \mathrm{MHz}) \delta_{\mathrm{C}} 15.668\left(\mathrm{CH}_{3}\right), 55.3$ $\left(\mathrm{CH}_{3}\right), 58.6\left(\mathrm{CH}_{3}\right), 59.1\left(\mathrm{CH}_{3}\right), 59.3\left(\mathrm{CH}_{3}\right), 59.4\left(\mathrm{CH}_{3}\right), 59.9\left(\mathrm{CH}_{2}\right)$, $60.0\left(\mathrm{CH}_{3}\right), 68.9(\mathrm{CH}), 69.3(\mathrm{CH}), 71.2(\mathrm{CH}), 77.7(\mathrm{CH}), 78.26$ $(\mathrm{CH}), 78.31(\mathrm{CH}), 79.212(\mathrm{CH}), 79.6(\mathrm{CH}), 98.3(\mathrm{CH}), 101.4(\mathrm{CH})$; MS (FAB) $m / z$ (\%) $433\left(\mathrm{M}^{+}+\mathrm{Na}, 88\right), 411$ (26), 133 (100); HRMS $\mathrm{m} / \mathrm{z}$ calcd for $\mathrm{C}_{18} \mathrm{H}_{34} \mathrm{NaO}_{10} 433.2050$, found 433.2051. Anal. Calcd for $\mathrm{C}_{18} \mathrm{H}_{34} \mathrm{O}_{10}$ : C, 52.67; H, 8.35. Found: C, 52.73; H, 8.06.

Method B with $\boldsymbol{n}$-Bu3 $S n D$. A solution of phthalimide 24 (43 $\mathrm{mg}, 0.078 \mathrm{mmol})$ in dry benzene $(5.8 \mathrm{~mL})$ containing $n-\mathrm{Bu}_{3} \mathrm{SnD}$ $(21 \mu \mathrm{L}, 0.078 \mathrm{mmol})$ and AIBN $(1.3 \mathrm{mg}, 0.008 \mathrm{mmol})$ was heated at reflux temperature for $1 \mathrm{~h}$. After this time another portion of $n$ $\mathrm{Bu}_{3} \mathrm{SnD}(21 \mu \mathrm{L}, 0.078 \mathrm{mmol})$ and $\mathrm{AIBN}(1.3 \mathrm{mg}, 0.008 \mathrm{mmol})$ were added and heating at reflux was continued for an additional $1 \mathrm{~h}$. After cooling to room temperature the reaction mixture was concentrated under reduced pressure. The residue was dissolved in $\mathrm{CH}_{3} \mathrm{CN}$, washed with $n$-hexane and the combined more polar extracts were concentrated under reduced pressure. Column chromatography (hexanes-EtOAc, 50:50 f 30:70) gave methyl 6-deoxy2,3,4-tri- $O$-acetyl- $\$$-D-(5-2H)gulopyranosyl-(1f4)-2,3-di- $O$-methylR-D-galactopyranoside [27-(D)] (16.6 mg, $0.040 \mathrm{mmol}, 52 \%)$, methyl 2,3,4-tri- $O$-methyl-R-L-[5-2H]rhamnopyranosyl-(1f4)-2,3- 
di- $O$-methyl-R-D-galactopyranoside (23-[D]) $(4.8 \mathrm{mg}, 0.012 \mathrm{mmol}$, $15 \%,{ }^{1} \mathrm{H} / 2 \mathrm{H}$ ratio $2: 8$ ) (contaminated with ca. $30 \%$ of compound 29), and methyl (5S)-5,6-anhydro-2,3-di- $O$-methyl-4,6-dideoxy-R-

D-erythro-hexos-5-ulopyranosyl-(1f4)-2,3-di-O-methyl-R-D-glucopyranoside (29) (3.6 mg, $0.010 \mathrm{mmol}, 12 \%$ ), all as colorless oils. Compound 27-(D): ${ }^{1} \mathrm{H}$ NMR $\delta_{\mathrm{H}} 1.24(3 \mathrm{H}, \mathrm{s}), 3.07(1 \mathrm{H}, \mathrm{d}, J) \quad 3.5$ $\mathrm{Hz}), 3.27(1 \mathrm{H}, \mathrm{dd}, J)$ 8.0, $3.0 \mathrm{~Hz}), 3.38(3 \mathrm{H}, \mathrm{s}), 3.45(3 \mathrm{H}, \mathrm{s})$, $3.47(3 \mathrm{H}, \mathrm{s}), 3.48(3 \mathrm{H}, \mathrm{s}), 3.49(3 \mathrm{H}, \mathrm{s}), 3.55(1 \mathrm{H}, \mathrm{m}), 3.57(1 \mathrm{H}$, $\mathrm{dd}, J) \quad 10.0,3.0 \mathrm{~Hz}), 3.60(3 \mathrm{H}, \mathrm{s}), 3.61(1 \mathrm{H}, \mathrm{dd}, J) \quad 10.0,3.0$ $\mathrm{Hz}), 3.72(1 \mathrm{H}, \mathrm{dd}, J) \quad 3.5,3.5 \mathrm{~Hz}), 3.74-3.82(2 \mathrm{H}, \mathrm{m}), 4.25(1 \mathrm{H}$, dd, $J$ ) 2.5, 0 Hz), $4.80(1 \mathrm{H}, \mathrm{d}, J) \quad 3.5 \mathrm{~Hz}), 4.90(1 \mathrm{H}, \mathrm{d}, J) 8.0$ $\mathrm{Hz}) ;{ }^{13} \mathrm{C} \mathrm{NMR} \delta_{\mathrm{C}} 15.541\left(\mathrm{CH}_{3}\right), 55.3\left(\mathrm{CH}_{3}\right), 58.6\left(\mathrm{CH}_{3}\right), 59.1$ $\left(\mathrm{CH}_{3}\right), 59.3\left(\mathrm{CH}_{3}\right), 59.4\left(\mathrm{CH}_{3}\right), 59.9\left(\mathrm{CH}_{2}\right), 60.0\left(\mathrm{CH}_{3}\right), 69.2(\mathrm{CH})$, $71.1(\mathrm{CH}), 77.6(\mathrm{CH}), 78.20(\mathrm{CH}), 78.26(\mathrm{CH}), 79.075(\mathrm{CH}), 79.6$ $(\mathrm{CH}), 98.3(\mathrm{CH}), 101.3(\mathrm{CH})$; MS (FAB) $\mathrm{m} / z(\%) 434\left(\mathrm{M}^{+}+\mathrm{Na}\right.$ 100), 133 (51); HRMS m/z calcd for $\mathrm{C}_{18} \mathrm{H}_{33}{ }^{2} \mathrm{HNaO}_{10} 434.2112$, found 434.2109. Compound 23-[D] (data taken from a mixture 23[D]/29, 7:3): ${ }^{1} \mathrm{H}$ NMR $(400 \mathrm{MHz}) \delta_{\mathrm{H}} 1.29(3 \mathrm{H}, \mathrm{s}), 3.13(1 \mathrm{H}, \mathrm{d}, J$ ) $9.3 \mathrm{~Hz}), 3.42(3 \mathrm{H}, \mathrm{s}), 3.46(1 \mathrm{H}, \mathrm{dd}, J) 9.3,2.7 \mathrm{~Hz}), 3.48(3 \mathrm{H}$, s), $3.51(6 \mathrm{H}, \mathrm{s}), 3.52(3 \mathrm{H}, \mathrm{s}), 3.55(3 \mathrm{H}, \mathrm{s}), 3.58(1 \mathrm{H}, \mathrm{dd}, J) \quad 2.1$, $2.1 \mathrm{~Hz}), 3.63-3.69(2 \mathrm{H}, \mathrm{m}), 3.75(1 \mathrm{H}, \mathrm{dd}, J) \quad 3.2,1.8 \mathrm{~Hz}), 3.78$ $(1 \mathrm{H}, \mathrm{dd}, J) \quad 11.1,7.2 \mathrm{~Hz}), 3.86(1 \mathrm{H}, \mathrm{ddd}, J) \quad 6.9,6.9,0 \mathrm{~Hz})$, $4.16(1 \mathrm{H}$, br s), $4.89(1 \mathrm{H}, \mathrm{d}, J) \quad 2.6 \mathrm{~Hz}), 5.11(1 \mathrm{H}, \mathrm{d}, J) \quad 1.9$ $\mathrm{Hz}) ;{ }^{13} \mathrm{C} \mathrm{NMR} \delta_{\mathrm{C}} 17.589\left(\mathrm{CH}_{3}\right), 17.648\left(\mathrm{CH}_{3}\right), 55.2\left(\mathrm{CH}_{3}\right), 57.6$ $\left(\mathrm{CH}_{3}\right), 58.48\left(\mathrm{CH}_{3}\right), 58.51\left(\mathrm{CH}_{3}\right), 58.61\left(\mathrm{CH}_{3}\right), 60.7\left(\mathrm{CH}_{3}\right), 61.7$ $\left(\mathrm{CH}_{2}\right), 69.8(\mathrm{CH}), 73.8(\mathrm{CH}), 77.3(\mathrm{CH}), 77.8(\mathrm{CH}), 79.8(\mathrm{CH})$, $80.5(\mathrm{CH}), 81.553(\mathrm{CH}), 81.628(\mathrm{CH}), 97.7(\mathrm{CH}), 99.1(\mathrm{CH}) ; \mathrm{MS}$ $m / z(\%) 411\left(\mathrm{M}^{+}+\mathrm{H}, 6\right), 393(17), 88(100)$. Compound 29: $[\mathrm{R}]_{\mathrm{D}}$ +103.3 (c, 0.12); IR 2930, 1104, $1053 \mathrm{~cm}^{-1} ;{ }^{1} \mathrm{H}$ NMR (400 MHz) $\left.\delta_{\mathrm{H}} 1.36(3 \mathrm{H}, \mathrm{s}), 1.93(1 \mathrm{H}, \mathrm{dd}, J) \quad 12.5,12.5 \mathrm{~Hz}\right), 2.09(1 \mathrm{H}, \mathrm{dd}, J$ ) $12.7,4.8 \mathrm{~Hz}), 3.39(3 \mathrm{H}, \mathrm{s}), 3.40(3 \mathrm{H}, \mathrm{s}), 3.46-3.54(2 \mathrm{H}, \mathrm{m})$, $3.47(3 \mathrm{H}, \mathrm{s}), 3.51(3 \mathrm{H}, \mathrm{s}), 3.52(3 \mathrm{H}, \mathrm{s}), 3.61(1 \mathrm{H}, \mathrm{dd}, J) \quad 10.0,3.7$ $\mathrm{Hz}), 3.74(1 \mathrm{H}, \mathrm{m}), 3.78(1 \mathrm{H}, \mathrm{dd}, J) \quad 13.5,2.4 \mathrm{~Hz}), 3.95(1 \mathrm{H}, \mathrm{ddd}$, $J) \quad 11.9,4.8,2.9 \mathrm{~Hz}), 4.08(1 \mathrm{H}, \mathrm{dd}, J) 13.5,1.3 \mathrm{~Hz}), 4.16(1 \mathrm{H}$, dd, $J) \quad 3.2,0 \mathrm{~Hz}), 4.95(1 \mathrm{H}, \mathrm{d}, J) \quad 3.7 \mathrm{~Hz}), 5.01(1 \mathrm{H}, \mathrm{d}, J) 1.8$ $\mathrm{Hz}) ;{ }^{13} \mathrm{C} \mathrm{NMR}(100.6 \mathrm{MHz}) \delta_{\mathrm{C}} 25.3\left(\mathrm{CH}_{3}\right), 35.6\left(\mathrm{CH}_{2}\right), 55.3\left(\mathrm{CH}_{3}\right)$, $56.0\left(\mathrm{CH}_{3}\right), 58.1\left(\mathrm{CH}_{3}\right), 58.7\left(\mathrm{CH}_{3}\right), 59.1\left(\mathrm{CH}_{3}\right), 63.2\left(\mathrm{CH}_{2}\right), 67.4$ $(\mathrm{CH}), 72.1(\mathrm{CH}), 73.5(\mathrm{CH}), 74.4(\mathrm{CH}), 77.3(\mathrm{CH}), 78.6(\mathrm{CH})$, $98.0(\mathrm{CH}), 98.7(\mathrm{CH}), 100.4(\mathrm{C})$; MS m/z (\%) $378\left(\mathrm{M}^{+}, 18\right), 347$ (11), 88 (100); HRMS m/z calcd for $\mathrm{C}_{17} \mathrm{H}_{30} \mathrm{O}_{9} 378.1890$, found 378.1886. Anal. Calcd for $\mathrm{C}_{17} \mathrm{H}_{30} \mathrm{O}_{9}$ : C, 53.96; H, 7.99. Found: C, 54.07; H, 7.97.

Methyl 2,3,4-Tri- $O$-acetyl-r-L-rhamnopyranosyl-(1f4)-2,3-di$\boldsymbol{O}$-methyl-6- $\boldsymbol{O}$-phthalimido-r-D-galactopyranoside (26). DEAD (278 $\mu \mathrm{L}, 1.77 \mathrm{mmol}$ ) was added dropwise to a stirred solution of the alcohol 25 (350 mg, $0.708 \mathrm{mmol}), N$-hydroxyphthalimide (388 mg, $1.77 \mathrm{mmol})$ and $\mathrm{PPh}_{3}(464 \mathrm{mg}, 1.77 \mathrm{mmol})$ in dry THF $(7.7 \mathrm{~mL})$ under nitrogen at $0{ }^{\circ} \mathrm{C}$ and the resulting solution was stirred at this temperature for $1.5 \mathrm{~h}$. The reaction was quenched with water and extracted with $\mathrm{CHCl}_{3}$. The combined extracts were dried over $\mathrm{Na}_{2} \mathrm{SO}_{4}$ and concentrated under reduced pressure. The residue obtained was purified by column chromatography (hexanes-EtOAc, $80: 20)$ to give $N$-phthalimide 26 (380 $\mathrm{mg}, 0.595 \mathrm{mmol}, 84 \%)$ as an amorphous solid: $[R]_{\mathrm{D}}+17.5(c, 0.245)$; IR $1791,1735 \mathrm{~cm}^{-1}$; ${ }^{1} \mathrm{H}$ NMR $\left.\delta_{\mathrm{H}} 1.15(3 \mathrm{H}, \mathrm{d}, J) \quad 6.3 \mathrm{~Hz}\right), 1.97(3 \mathrm{H}, \mathrm{s}), 2.04(3 \mathrm{H}, \mathrm{s})$, $2.12(3 \mathrm{H}, \mathrm{s}), 3.44(3 \mathrm{H}, \mathrm{s}), 3.48(3 \mathrm{H}, \mathrm{s}), 3.52(3 \mathrm{H}, \mathrm{s}), 3.59(1 \mathrm{H}, \mathrm{dd}$, $J$ ) $10.1,2.7 \mathrm{~Hz}), 3.66(1 \mathrm{H}, \mathrm{dd}, J) 10.1,3.5 \mathrm{~Hz}), 3.95(1 \mathrm{H}$, dddd, $J) \quad 9.9,6.3,6.3,6.3 \mathrm{~Hz}), 4.13(1 \mathrm{H}, \mathrm{ddd}, J) \quad 6.1,6.1,0 \mathrm{~Hz}), 4.33$ $(1 \mathrm{H}, \mathrm{dd}, J) \quad 11.1,5.9 \mathrm{~Hz}), 4.36(1 \mathrm{H}, \mathrm{dd}, J) \quad 11.1,6.2 \mathrm{~Hz}), 4.40$ $(1 \mathrm{H}, \mathrm{dd}, J) \quad 1.1,0 \mathrm{~Hz}), 4.87(1 \mathrm{H}, \mathrm{d}, J) \quad 3.5 \mathrm{~Hz}), 5.05(1 \mathrm{H}, \mathrm{dd}$, $J$ ) $9.9,9.9 \mathrm{~Hz}), 5.07(1 \mathrm{H}, \mathrm{d}, J) \quad 1.5 \mathrm{~Hz}), 5.29(1 \mathrm{H}, \mathrm{dd}, J) \quad 10.1$, $3.3 \mathrm{~Hz}), 5.50(1 \mathrm{H}, \mathrm{dd}, J) \quad 3.0,2.1 \mathrm{~Hz}), 7.76(2 \mathrm{H}, \mathrm{m}), 7.84(2 \mathrm{H}$, m); ${ }^{13} \mathrm{C} \mathrm{NMR}(100.6 \mathrm{MHz}) \delta_{\mathrm{C}} 17.3\left(\mathrm{CH}_{3}\right), 20.7\left(\mathrm{CH}_{3}\right), 20.8\left(\mathrm{CH}_{3}\right)$, $20.9\left(\mathrm{CH}_{3}\right), 55.8\left(\mathrm{CH}_{3}\right), 58.6\left(\mathrm{CH}_{3}\right), 59.3\left(\mathrm{CH}_{3}\right), 67.3(\mathrm{CH}), 67.6$ $(\mathrm{CH}), 69.1(\mathrm{CH}), 69.9(\mathrm{CH}), 70.8(\mathrm{CH}), 75.0(\mathrm{CH}), 77.4\left(\mathrm{CH}_{2}\right)$, $77.6(\mathrm{CH}), 79.6(\mathrm{CH}), 98.4(\mathrm{CH}), 99.3(\mathrm{CH}), 123.6(2 \times \mathrm{CH}), 128.8$ $(2 \times \mathrm{C}), 134.6(2 \times \mathrm{CH}), 163.5(2 \times \mathrm{C}), 169.8(\mathrm{C}), 169.9(\mathrm{C})$, $170.0(\mathrm{C})$; MS (FAB) $\mathrm{m} / \mathrm{z}(\%) 663\left(\mathrm{M}^{+}+\mathrm{H}+\mathrm{Na}, 3\right), 662(9)$, 273 (28), 55 (100); HRMS calcd for $\mathrm{C}_{29} \mathrm{H}_{38} \mathrm{NNaO}_{15} 663.2139$, found
663.2166. Anal. Calcd for $\mathrm{C}_{29} \mathrm{H}_{37} \mathrm{NO}_{15}$ : C, 54.46; H, 5.83; N, 2.19. Found: C, 54.10; H, 5.78; N, 2.38.

Reductive HAT of Methyl 2,3,4-Tri- $O$-acetyl-r-L-rhamnopyranosyl-(1f4)-2,3-di- $O$-methyl-6- $O$-phthalimido-r-D-galactopyranoside (26). Method A with $\boldsymbol{n}$-Bu $\mathrm{Bu}_{3} \mathrm{SnH}$. A solution of phthalimide 26 (95 $\mathrm{mg}, 0.149 \mathrm{mmol})$ in dry benzene $(11.2 \mathrm{~mL})$ containing $n-\mathrm{Bu}_{3} \mathrm{SnH}(40 \mu \mathrm{L}, 0.149 \mathrm{mmol})$ and $\mathrm{AIBN}(2.4 \mathrm{mg}, 0.015 \mathrm{mmol})$ was heated at reflux temperature for $1.5 \mathrm{~h}$. After cooling to room temperature the reaction mixture was concentrated under reduced pressure. The residue was dissolved in $\mathrm{CH}_{3} \mathrm{CN}$, washed with $n$ hexane and the combined more polar extracts were concentrated under reduced pressure. The residue was purified by column chromatography (hexanes-EtOAc, 50:50 f 30:70) to give methyl 2,3-di-O-acetyl-4,6-dideoxy-\$-D-erythro-hex-4-enopyranosyl-(1 f4)2,3-di- $O$-methyl-R-D-galactopyranoside (30) $(3.5 \mathrm{mg}, 0.008 \mathrm{mmol}$, $5 \%$ ), the alcohol 25 (16.5 $\mathrm{mg}, 0.033 \mathrm{mmol}, 22 \%)$, previously described, and the methyl 2,3,4-tri- $O$-acetyl-6-deoxy- $\S$-D-gulopyranosyl-(1f4)-2,3-di-O-methyl-R-D-galactopyranoside (28) (38.2 $\mathrm{mg}, 0.077 \mathrm{mmol}, 52 \%$ ) as colorless oils. Compound 30: $[\mathrm{R}]_{\mathrm{D}}-33.8$ $(c, 0.29)$; IR 3468, $1747 \mathrm{~cm}^{-1} ;{ }^{1} \mathrm{H}$ NMR $(400 \mathrm{MHz}) \delta_{\mathrm{H}} 1.83(3 \mathrm{H}$, s), $2.05(3 \mathrm{H}, \mathrm{s}), 2.10(3 \mathrm{H}, \mathrm{s}), 2.21(1 \mathrm{H}, \mathrm{dd}, J) \quad 9.8,7.5 \mathrm{~Hz}), 3.41$ $(3 \mathrm{H}, \mathrm{s}), 3.50(3 \mathrm{H}, \mathrm{s}), 3.51(3 \mathrm{H}, \mathrm{s}), 3.57-3.58(2 \mathrm{H}, \mathrm{m}), 3.60-3.75$ $(2 \mathrm{H}, \mathrm{m}), 3.82(1 \mathrm{H}, \mathrm{ddd}, J) \quad 6.6,6.6,1.3 \mathrm{~Hz}), 4.24(1 \mathrm{H}, \mathrm{dd}, J)$ $1.1,1.1 \mathrm{~Hz}), 4.66(1 \mathrm{H}$, br d, $J) 3.7 \mathrm{~Hz}), 4.85(1 \mathrm{H}, \mathrm{d}, J) 1.6 \mathrm{~Hz})$, $5.25(1 \mathrm{H}, \mathrm{dd}, J) \quad 5.0,5.0 \mathrm{~Hz}), 5.30(1 \mathrm{H}, \mathrm{d}, J) 5.3 \mathrm{~Hz}), 5.51(1 \mathrm{H}$, ddd, $J) \quad 5.3,3.7,1.6 \mathrm{~Hz}) ;{ }^{13} \mathrm{C}$ NMR $(100.6 \mathrm{MHz}) \delta_{\mathrm{C}} 19.5\left(\mathrm{CH}_{3}\right)$, $20.8\left(\mathrm{CH}_{3}\right), 20.9\left(\mathrm{CH}_{3}\right), 55.4\left(\mathrm{CH}_{3}\right), 58.7\left(\mathrm{CH}_{3}\right), 59.1\left(\mathrm{CH}_{3}\right), 61.4$ $\left(\mathrm{CH}_{2}\right), 64.1(\mathrm{CH}), 66.0(\mathrm{CH}), 69.6(\mathrm{CH}), 73.4(\mathrm{CH}), 78.0(\mathrm{CH})$, $79.6(\mathrm{CH}), 95.1(\mathrm{CH}), 97.9(\mathrm{CH}), 98.4(\mathrm{CH}), 151.1(\mathrm{C}), 169.9(\mathrm{C})$, 170.2 (C); MS m/z (\%) $435\left(\mathrm{M}^{+}+\mathrm{H},<1\right), 402(<1), 374$ (1), 212 (11), 88 (100); HRMS calcd for $\mathrm{C}_{19} \mathrm{H}_{31} \mathrm{O}_{11}$ 435.1866, found 435.1877. Anal. Calcd for $\mathrm{C}_{19} \mathrm{H}_{30} \mathrm{O}_{11}$ : C, 52.53; H, 6.96. Found: C, 52.23; H, 6.90. Compound 28: crystalline solid, mp 153.2-154.9 ${ }^{\circ} \mathrm{C}$ (from $n$-hexane-acetone); $[\mathrm{R}]_{\mathrm{D}}+60.0(c, 0.53) ; \mathrm{IR} 3506,1748$ $\mathrm{cm}^{-1} ;{ }^{1} \mathrm{H}$ NMR $\left.\delta_{\mathrm{H}} 1.19(3 \mathrm{H}, \mathrm{d}, J) \quad 6.4 \mathrm{~Hz}\right), 2.03(3 \mathrm{H}, \mathrm{s}), 2.13$ $(3 \mathrm{H}, \mathrm{s}), 2.17(3 \mathrm{H}, \mathrm{s}), 3.23(1 \mathrm{H}, \mathrm{m}), 3.39(3 \mathrm{H}, \mathrm{s}), 3.47(1 \mathrm{H}, \mathrm{dd}, J)$ $10.1,3.5 \mathrm{~Hz}), 3.48(3 \mathrm{H}, \mathrm{s}), 3.49(3 \mathrm{H}, \mathrm{s}), 3.57(1 \mathrm{H}, \mathrm{dd}, J) 10.1$, $3.0 \mathrm{~Hz}), 3.63(1 \mathrm{H}, \mathrm{m}), 3.77-3.83(2 \mathrm{H}, \mathrm{m}), 4.15(1 \mathrm{H}, \mathrm{dddd}, J)$ $6.5,6.5,6.5,1.3 \mathrm{~Hz}), 4.19(1 \mathrm{H}, \mathrm{br} \mathrm{d}, J) 3.1 \mathrm{~Hz}), 4.81(1 \mathrm{H}, \mathrm{d}, J$ ) $3.5 \mathrm{~Hz}), 4.83(1 \mathrm{H}, \mathrm{dd}, J) \quad 3.7,1.4 \mathrm{~Hz}), 4.94(1 \mathrm{H}, \mathrm{d}, J) \quad 8.3$ $\mathrm{Hz}), 5.04(1 \mathrm{H}, \mathrm{dd}, J)$ 8.3, $3.5 \mathrm{~Hz}), 5.34(1 \mathrm{H}, \mathrm{dd}, J)$ 3.6, 3.6 $\mathrm{Hz}) ;{ }^{13} \mathrm{C}$ NMR $(100.6 \mathrm{MHz}) \delta_{\mathrm{C}} 15.715\left(\mathrm{CH}_{3}\right), 20.6\left(\mathrm{CH}_{3}\right), 20.7$ $\left(\mathrm{CH}_{3}\right), 20.8\left(\mathrm{CH}_{3}\right), 55.4\left(\mathrm{CH}_{3}\right), 58.4\left(\mathrm{CH}_{3}\right), 59.1\left(\mathrm{CH}_{3}\right), 60.2\left(\mathrm{CH}_{2}\right)$, $67.9(\mathrm{CH}), 68.4(\mathrm{CH}), 68.8(\mathrm{CH}), 69.0(\mathrm{CH}), 70.100(\mathrm{CH}), 73.3$ $(\mathrm{CH}), 78.0(\mathrm{CH}), 79.2(\mathrm{CH}), 97.9(\mathrm{CH}), 99.8(\mathrm{CH}), 168.9(\mathrm{C}), 169.5$ (C), 169.8 (C); MS (FAB) $\mathrm{m} / \mathrm{z}(\%) 518\left(\mathrm{M}^{+}+\mathrm{H}+\mathrm{Na}, 6\right), 517$ (21), 391 (32), 273 (63), 73 (100); HRMS calcd for $\mathrm{C}_{21} \mathrm{H}_{35} \mathrm{NaO}_{13}$ 518.1975, found 518.1984. Anal. Calcd for $\mathrm{C}_{21} \mathrm{H}_{34} \mathrm{O}_{13}$ : C, 51.01; H, 6.93. Found: C, 51.15; H, 6.89 .

Method B with $\boldsymbol{n}$-Bu $\mathbf{u}_{3} \mathrm{SnD}$. A solution of phthalimide 26 (90 $\mathrm{mg}, 0.141 \mathrm{mmol})$ in dry benzene $(10.6 \mathrm{~mL})$ containing $n-\mathrm{Bu}_{3} \mathrm{SnD}$ $(38 \mu \mathrm{L}, 0.141 \mathrm{mmol})$ and AIBN $(2.3 \mathrm{mg}, 0.014 \mathrm{mmol})$ was heated at reflux temperature for $1 \mathrm{~h}$. After this time another portion of $n$ $\mathrm{Bu}_{3} \mathrm{SnD}(38 \mu \mathrm{L}, 0.141 \mathrm{mmol})$ and $\operatorname{AIBN}(2.3 \mathrm{mg}, 0.014 \mathrm{mmol})$ were added and heating at reflux was continued for an additional $1 \mathrm{~h}$. After cooling to room temperature the reaction mixture was concentrated under reduced pressure. The residue was dissolved in $\mathrm{CH}_{3} \mathrm{CN}$, washed with $n$-hexane and the combined more polar extracts were concentrated under reduced pressure. The residue was purified by column chromatography (hexanes-EtOAc, 50:50 f $30: 70)$ to give the olefin $30(9 \mathrm{mg}, 0.021 \mathrm{mmol}, 15 \%)$, described above, methyl 2,3,4-tri- $O$-acetyl-R-L-[5-2H]rhamnopyranosyl-(1f4)2,3-di- $O$-methyl-R-D-galactopyranoside (25-[D]) (12.6 mg, 0.025 mmol, $18 \%,{ }^{1} \mathrm{H} /{ }^{2} \mathrm{H}$ ratio, 3:7), and methyl 2,3,4-tri- $O$-acetyl-6deoxy-§-D-(5-2 H)gulopyranosyl-(1f4)-2,3-di-O-methyl-R-D-galactopyranoside [28-(D)] (31.2 $\mathrm{mg}, 0.063 \mathrm{mmol}, 45 \%)$ as colorless oils. Compound 25-[D]: ${ }^{1} \mathrm{H}$ NMR $\delta_{\mathrm{H}} 1.22(3 \mathrm{H}, \mathrm{s}), 1.23(3 \mathrm{H}, \mathrm{d}, J$ ) $6.6 \mathrm{~Hz}), 1.99(3 \mathrm{H}, \mathrm{s}), 2.05(3 \mathrm{H}, \mathrm{s}), 2.14(3 \mathrm{H}, \mathrm{s}), 3.42(3 \mathrm{H}, \mathrm{s})$, $3.47(3 \mathrm{H}, \mathrm{s}), 3.54(3 \mathrm{H}, \mathrm{s}), 3.56(1 \mathrm{H}, \mathrm{dd}, J) \quad 10.1,2.9 \mathrm{~Hz}), 3.64$ 
$(1 \mathrm{H}, \mathrm{dd}, J)$ 10.1, $3.5 \mathrm{~Hz}), 3.66(1 \mathrm{H}, \mathrm{m}), 3.80-3.86(2 \mathrm{H}, \mathrm{m}), 3.98$ $(1 \mathrm{H}$, dddd, $J) \quad 10.0,6.3,6.3,6.3 \mathrm{~Hz}), 4.12(1 \mathrm{H}, \mathrm{dd}, J) \quad 2.7,0$ $\mathrm{Hz}), 4.88(1 \mathrm{H}, \mathrm{d}, J) \quad 3.5 \mathrm{~Hz}), 5.05(1 \mathrm{H}, \mathrm{d}, J) 2.1 \mathrm{~Hz}), 5.071$ $(1 \mathrm{H}, \mathrm{d}, J) \quad 10.0 \mathrm{~Hz}), 5.073(1 \mathrm{H}, \mathrm{dd}, J) \quad 9.8,9.8 \mathrm{~Hz}), 5.31(1 \mathrm{H}$, dd, $J) \quad 10.0,3.3 \mathrm{~Hz}), 5.47(1 \mathrm{H}, \mathrm{dd}, J) \quad 3.3,2.1 \mathrm{~Hz}) ;{ }^{13} \mathrm{C}$ NMR (100.6 MHz) $\delta_{\mathrm{C}} 17.350\left(\mathrm{CH}_{3}\right), 17.489\left(\mathrm{CH}_{3}\right), 20.7\left(\mathrm{CH}_{3}\right), 20.8$ $\left(\mathrm{CH}_{3}\right), 20.9\left(\mathrm{CH}_{3}\right), 55.4\left(\mathrm{CH}_{3}\right), 58.8\left(\mathrm{CH}_{3}\right), 59.3\left(\mathrm{CH}_{3}\right), 62.0\left(\mathrm{CH}_{2}\right)$, $67.5(\mathrm{CH}), 69.0(\mathrm{CH}), 69.8(\mathrm{CH}), 69.9(\mathrm{CH}), 70.869(\mathrm{CH}), 70.932$ $(\mathrm{CH}), 75.1(\mathrm{CH}), 78.0(\mathrm{CH}), 79.8(\mathrm{CH}), 98.1(\mathrm{CH}), 99.7(\mathrm{CH})$, 169.8 (C), 170.0 (C), 170.0 (C); MS (FAB) m/z (\%) $519\left(\mathrm{M}^{+}+\right.$ $\mathrm{Na}+\mathrm{H}, 7), 518$ (26), 517 (5), 274 (46), 273 (27), 73 (100); HRMS calcd for $\mathrm{C}_{21} \mathrm{H}_{34}{ }^{2} \mathrm{HNaO}_{13}$ 519.2038, found 519.2042. Compound 28-(D): ${ }^{1} \mathrm{H}$ NMR $\delta_{\mathrm{H}} 1.18(3 \mathrm{H}, \mathrm{s}), 2.02(3 \mathrm{H}, \mathrm{s}), 2.12(3 \mathrm{H}, \mathrm{s}), 2.16$ $(3 \mathrm{H}, \mathrm{s}), 3.24(1 \mathrm{H}, \mathrm{m}), 3.38(3 \mathrm{H}, \mathrm{s}), 3.46(1 \mathrm{H}, \mathrm{dd}, J) 10.1,3.5$ $\mathrm{Hz}), 3.47(3 \mathrm{H}, \mathrm{s}), 3.48(3 \mathrm{H}, \mathrm{s}), 3.56(1 \mathrm{H}, \mathrm{dd}, J) \quad 10.1,3.0 \mathrm{~Hz})$, $3.63(1 \mathrm{H}, \mathrm{m}), 3.76-3.82(2 \mathrm{H}, \mathrm{m}), 4.19(1 \mathrm{H}, \mathrm{dd}, J) 3.0,0 \mathrm{~Hz})$, $4.80(1 \mathrm{H}, \mathrm{d}, J) \quad 3.5 \mathrm{~Hz}), 4.81(1 \mathrm{H}, \mathrm{d}, J) \quad 3.7 \mathrm{~Hz}), 4.94(1 \mathrm{H}, \mathrm{d}$, $J) 8.3 \mathrm{~Hz}), 5.03(1 \mathrm{H}, \mathrm{dd}, J) \quad 8.3,3.5 \mathrm{~Hz}), 5.33(1 \mathrm{H}, \mathrm{dd}, J) \quad 3.6$, $3.6 \mathrm{~Hz}) ;{ }^{13} \mathrm{C} \mathrm{NMR}(100.6 \mathrm{MHz}) \delta_{\mathrm{C}} 15.576\left(\mathrm{CH}_{3}\right), 20.6\left(\mathrm{CH}_{3}\right), 20.7$ $\left(\mathrm{CH}_{3}\right), 20.7\left(\mathrm{CH}_{3}\right), 55.4\left(\mathrm{CH}_{3}\right), 58.4\left(\mathrm{CH}_{3}\right), 59.0\left(\mathrm{CH}_{3}\right), 60.2\left(\mathrm{CH}_{2}\right)$,
$67.9(\mathrm{CH}), 68.4(\mathrm{CH}), 69.0(\mathrm{CH}), 70.036(\mathrm{CH}), 73.3(\mathrm{CH}), 78.0$ (CH), $79.2(\mathrm{CH}), 97.9(\mathrm{CH}), 99.8(\mathrm{CH}), 168.8(\mathrm{C}), 169.5(\mathrm{C}), 169.8$ (C); MS (FAB) $m / z(\%) 519\left(\mathrm{M}^{+}+\mathrm{H}+\mathrm{Na}, 2\right), 518$ (7), 355 (10), 274 (27), 73 (100); HRMS calcd for $\mathrm{C}_{2} \mathrm{H} 33 \mathrm{HNaO} 519.2038$, found 519.2014.

Acknowledgment. This work was supported by Research programs CTQ2004-06381/BQU, CTQ2004-02367/BQU, and CTQ2007-67492/BQU of the Ministerio de Educacio'n y Ciencia, Spain, cofinanced by the Fondo Europeo de Desarrollo Regional (FEDER). I.P.-M. and L.M.Q. thank the I3P-CSIC Program for fellowships.

Supporting Information Available: A complete description of experimental details of precursors and copies of NMR spectra $\left({ }^{1} \mathrm{H}\right.$ and $\left.{ }^{13} \mathrm{C}\right)$ for all new compounds. This material is available free of charge via the Internet at http://pubs.acs.org.

JO801499D 